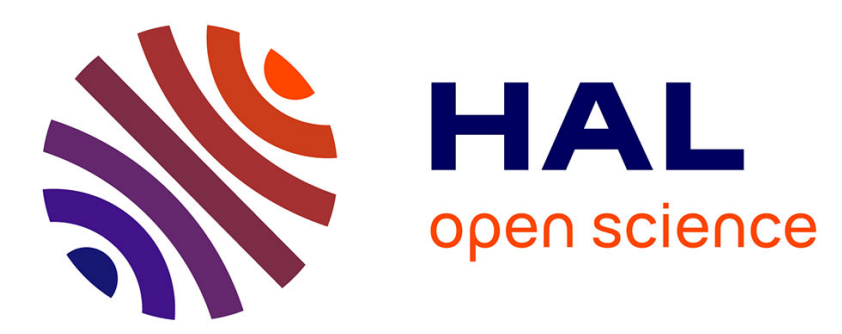

\title{
Variability and singularity arising from poor compliance in a pharmacokinetic model II: the multi-oral case
}

\author{
Lisandro J. Fermin, Jacques Lévy Véhel
}

\section{To cite this version:}

Lisandro J. Fermin, Jacques Lévy Véhel. Variability and singularity arising from poor compliance in a pharmacokinetic model II: the multi-oral case. Journal of Mathematical Biology, 2016, 10.1007/s00285-016-1041-1 . hal-00868621

\section{HAL Id: hal-00868621 \\ https://inria.hal.science/hal-00868621}

Submitted on 1 Oct 2013

HAL is a multi-disciplinary open access archive for the deposit and dissemination of scientific research documents, whether they are published or not. The documents may come from teaching and research institutions in France or abroad, or from public or private research centers.
L'archive ouverte pluridisciplinaire HAL, est destinée au dépôt et à la diffusion de documents scientifiques de niveau recherche, publiés ou non, émanant des établissements d'enseignement et de recherche français ou étrangers, des laboratoires publics ou privés. 


\title{
Variability and singularity arising from poor compliance in a pharmacokinetic model II: the multi-oral case
}

\author{
L.J. Fermín ${ }^{1,2}$ and J. Lévy Véhel ${ }^{2}$ \\ ${ }^{1}$ CIMFAV, Faculty of Engineering, University of Valparaiso, Valparaiso, Chile. \\ ${ }^{2}$ Regularity Team, INRIA Saclay-Ile-de-France \& MAS Laboratory, Ecole Centrale Paris, France.
}

\begin{abstract}
We study a simplified model for the drug concentration in the case of multiple oral doses and in a situation of poor patient compliance. Our model is a stochastic one, which is able to take into account an irregular drug intake schedule. This article is the second in a series of three. It presents a multi-oral version of the results given in [11], that dealt with the multi IV case. Under some assumptions, we study features of the drug concentration that have practical implications, such as its variability and the regularity of its probability distribution. We consider four variants: continuous-time, with either deterministic or random doses, and discrete-time, also with either deterministic or random doses. Our computations allow one to assess in a precise way the effect of various significant parameters such as the mean rate of intake, the elimination rate, the absorption rate and the mean dose. They quantify how much poor compliance will affect the regimen. To appreciate this impact, we provide detailed comparisons with the variability of concentration in the cases of both a fully compliant patient and a population of fully compliant patients with log-normaly distributed pharmacokinetic parameters. Besides, the discrete-time versions of our models reveal unexpected links with possibly multifractal measures known in mathematics as infinite Bernoulli convolutions. Analogously to the multi-IV case, we find that the distribution of the concentration in this model is either absolutely continuous or purely singular, depending on a relevant parameter. Our results complement the ones in [11] and help understanding the consequences of poor compliance. They may have practical outcomes in terms of drug dosing and scheduling.
\end{abstract}




\section{Contents}

1 Introduction $\quad 3$

2 Overview: pharmacokinetic implication of non compliance 4

2.1 Purely deterministic model (full compliance) . . . . . . . . . . . . . 4

2.2 Full compliance with population parameter variability . . . . . . . . . 7

2.3 Continuous model with random intake instants and deterministic doses . . . 10

2.4 Continuous model with random intake instants and random doses . . . . . . 16

2.5 Discrete model with random intake instants and deterministic doses . . . . 18

2.6 Discrete model with random intake instants and random doses . . . . . . . 19

3 Continuous model with random intake instants and deterministic doses 20

3.1 Variability of the concentration . . . . . . . . . . . . . . 21

3.2 Regularity of the limit distribution $\ldots \ldots \ldots 22$

4 Continuous model with random intake instants and random doses 25

4.1 Variability of the concentration . . . . . . . . . . . . . 25

4.2 Regularity of the limit distribution $\ldots \ldots \ldots . \ldots \ldots$

5 Discrete model with random intake instants and deterministic doses 29

5.1 The discretized concentration . . . . . . . . . . . . . . . . . . . 29

5.2 Variability of the discretized concentration . . . . . . . . . . . . 31

5.3 Limit when the discretization step tends to $0 \ldots \ldots \ldots 32$

5.4 Regularity of the discretized model . . . . . . . . . . . . . . 32

6 Discrete model with random intake instants and random doses 33

6.1 Variability of the concentration . . . . . . . . . . . . . . . 34

6.2 Limit when the discretization step tends to $0 \ldots \ldots$. . . . . . . . . 34 


\section{Introduction}

In the seminal work [13], the authors attacked the problem of mathematically modeling poor compliance using a probabilistic frame. They considered general distributions for the random instants of drug intake and studied the mean and variance of the concentration conditioned on the time elapsed since the last intake. Various related models were studied in detail in [11]: the probability distribution of drug concentration in the context of multipleIV dosing and poor compliance was investigated when both the times of intakes and doses are supposed to be random. In addition, both continuous-time and discrete-time versions were considered.

The present work parallels the studies of [11] in the more realistic framework of multiple oral dosing. As in [11], we investigate below the probability distribution of drug concentration in the context of poor compliance: the instants of drug intake are randoms, with possibly random doses. We use the simplest possible law to model the random times of drug intake, i.e. a homogeneous Poisson distribution. In other words, the times of drug intake are supposed to follows a Poisson process. This assumption allows one to perform explicit computations using the well-developed machinery on Poisson processes, and to obtain precise results describing various aspects of the distribution of the concentration that are important for assessing the efficacy of the regimen. We focus on two aspects of practical relevance: the variability of the concentration and the regularity of its probability distribution.

Our results quantify the variability of the concentration around its mean showing the exact role played by each parameter of the process. We measure how much poor compliance increases this variability as compared to the full compliance case. We show that the probability distribution limit of drug concentration may display irregular behaviours: in our case this occurs when the ratio between the mean number of intakes per unit time and the minimum between the elimination rate and the absorption rate is smaller than one half. When the same ratio is smaller than one, it becomes singular (i.e. non differentiable) at the origin. In practical terms, this amounts to quantifying, in a precise way, the situations where the moments of intakes are too scarce (with respect to the elimination and absorption rates), resulting in a high probability of having too small a concentration of drugs.

We also study a discrete-time version of the model. This setting reveals unexpected links with multifractal measures which have been studied in the mathematical literature for over seventy years under the name of infinite Bernoulli convolutions. Again, depending on some parameters, the discretized concentration may exhibit an extremely irregular behaviour. This means that the probability of observing a concentration $C$ depends in a very non-smooth way on the precise value of $C$. This is obviously an undesirable feature which may have strongly negative consequences.

As mentioned above, the present article is the second in a series of three, where the first work [11] dealt with the multi IV case. Since some computations here are very similar to this case, they are omitted and the interested reader is referred to [11] for full details. The third paper in the series, [4], extends our results by considering more realistic random 
schedules for the time instants of drug intakes: indeed, the use a homogeneous Poisson law is mathematically handy, but somewhat restrictive: in real situations, the schedule is probably much more complex. [4] uses the powerful mathematical framework known as Piecewise Deterministic Markov Model to deal with general drug intake schedules, at the expense of a more complex theoretical apparatus.

We do not address in this work the problem of estimating the parameters of the various models, in particular the law of the random intake times and doses. A recent article dealing with this issue is [2].

The remaining of this work is organized as follows: for the reader's convenience, we present an overview of our main findings pertaining to the various models and their practical implications in Section 2. In particular, we compare the variabilities in our poor compliance models to the ones in the cases of (a) full compliance of a single patient and (b) full compliance in a population where one takes into account variability due to differing elimination rates or clearances between individuals. This allows us to highlight "equivalent scenarios" where, for instance, we find the parameters of a non-compliant patient that will yield the same variance in concentration as in a whole population with given distribution of elimination rates, or, which is the same, of a single individual whose elimination rate is unknown and is modelled as a random variable. A reader not interested in the mathematical details may concentrate on Section 2 to get a quick summary of our work, and refer if needed to the details in the following sections. In Section 3, we set up the basic continuous time model for deterministic dose random time drug intake and study its variability (Section 3.1) and regularity (Section 3.2). In Section 4, we analyse the random doses version of this model. As before, we study its variability (Section 4.1) and regularity (Section 4.2). We present the discrete-time version of the deterministic dose random time model in Section 5. We derive the discrete time concentration in Section 5.1 and study its variability in Section 5.2. In Section 5.3, we show that the discrete-time model tends to the continuous-time one when the discretization step tends to 0 . Section 5.4 describes the complex regularity behaviour of the probability distribution of the concentration in the discrete-time model. Finally, Section 6 deals with the discrete-time random-dose case: its variability is described in Section 6.1, and its limiting behaviour when the discretization step tends to 0 is studied in Section 6.2.

\section{Overview: pharmacokinetic implication of non com- pliance}

\subsection{Purely deterministic model (full compliance)}

To assess the impact of poor compliance, it is useful to contrast it with situations of full compliance. We derive in this section the variability in concentration for a single fully compliant patient. The next section will deal with the variability in a population of fully compliant patients. 
Let us first recall the basic equations. We consider in this work the case of multiples oral administration and we suppose that kinetics of first order are involved. Our first model is a very classical one: it assumes that the patient takes orally a constant dose $D$ at regularly spaced times $t_{0}, t_{1}, \ldots, t_{n}, \ldots$, with $t_{i}=\frac{i}{\lambda}$, that is, the patient takes drugs every $\frac{1}{\lambda}$ units of times for some positive $\lambda$.

For completeness and future use, we briefly recall how the equations governing the absorption and elimination processes are obtained. The oral absorption process is concerned with the amount of drug at the absorption site remaining to be absorbed. The amount of drug at the absorption site, denoted $A_{\text {oral }}$, is characterized by the absorption coefficient rate $k_{a}$. At each drug intake time $t_{i}, A_{\text {oral }}(t)$ increases by $D$. Between $t_{i}$ and $t_{i+1}$, the effect of the dose taken at $t_{i}$ decreases exponentially fast, with exponential speed $k_{a}$. Formally:

$$
\frac{d}{d t} A_{\text {oral }}(t)=\sum_{i: t_{i} \leq t} D \delta\left(t-t_{i}\right)-k_{a} A_{\text {oral }}(t)
$$

with $\delta$ denoting the Dirac distribution. Thus, the rate of absorption of drug at time $t$ is $k_{a} F A_{\text {oral }}(t)$, where $F$ is the absolute bioavailability; i.e. the fraction of each dose which is absorbed when the drug is given by the oral route.

The elimination process describes by the irreversible loss of drug from the site of measurement. We assume that the drug is eliminated with a constant elimination rate $k_{e}$. Denoting $A_{\text {central }}$ the amount of drug in the body, one gets that the rate of elimination of drug is $k_{e} A_{\text {central }}$.

The model is given from mass balance considerations: at any given time, the dose is accounted for by the amount of drug at absorption site plus the amount of drug in body plus the amount of eliminated drug. Thus, the sum of the rates of change of the drug in these compartments must equal zero, so that the rate of change of drug in body is equal to the rate of absorption minus the rate of elimination. This leads to:

$$
\frac{d}{d t} A_{\text {central }}(t)=k_{a} F A_{\text {oral }}(t)-k_{e} A_{\text {central }}(t) .
$$

The drug concentration at time $t$ is defined by

$$
C(t)=\frac{A_{\text {central }}(t)}{V_{d}}
$$

where $V_{d}$ is the apparent volume of distribution of the drug with respect to its concentration in plasma.

The solution of equation (1) is

$$
A_{\text {oral }}(t)=D \sum_{i} e^{-k_{a}\left(t-t_{i}\right)} \mathrm{1}_{\left(t \geq t_{i}\right)},
$$

where $\mathbb{1}_{\left(t \geq t_{i}\right)}$ denotes the indicator function of the set $\left.\left\{t \in \mathbb{R}: t \geq t_{i}\right)\right\}$, which equals 1 if $t \geq t_{i}$ and 0 otherwise. 
Applying the parameter-variation method to equation (2) one obtains, from equations (3) and (4), the following expression for the deterministic drug concentration process $C_{d}$ :

$$
C_{d}(t)=\frac{F D}{V_{d}} \frac{k_{a}}{k_{a}-k_{e}} \sum_{i}\left(e^{-k_{e}\left(t-t_{i}\right)}-e^{-k_{a}\left(t-t_{i}\right)}\right) \mathbb{1}_{\left(t \geq t_{i}\right)} .
$$

We will wish to compare the values of the expectation and the variance in various stochastic models to the one in the case of full compliance. Of course, in the frame of full compliance, there is no randomness involved, and one cannot define proper mean and variance. However, since the concentration varies in time, it makes sense to define the mean as the average of concentration over all values of $t$, and to define the variance correspondingly. In other words, we denote:

$$
\begin{gathered}
E_{d}=\lim _{T \rightarrow \infty} \frac{1}{T} \int_{0}^{T} C_{d}(t) d t \\
\operatorname{Var}_{d}=\lim _{T \rightarrow \infty} \frac{1}{T} \int_{0}^{T}\left(C_{d}(t)-E_{d}\right)^{2} d t
\end{gathered}
$$

for the mean and variance with respect to time of $C_{d}(t)$. Note that $E_{d}$ is closely related to the usual PK metric $A U C$. $V a r_{d}$ represent the time-average square deviation from the long term average $E_{d}$. Simple computations lead to:

$$
\begin{gathered}
E_{d}=\mu_{e} \frac{F D}{V_{d}} \\
V a r_{d}=\frac{\mu_{e}}{2}\left(\frac{F D}{V_{d}}\right)^{2}\left[\frac{1}{1+r}+G(r)\right] .
\end{gathered}
$$

where

$$
G(r)=\frac{2}{1-r^{2}}\left(\frac{1}{e^{1 / \mu_{e}}-1}-\frac{r}{e^{1 / r \mu_{e}}-1}\right)-2 \mu_{e},
$$

$\mu_{e}:=\frac{\lambda}{k_{e}}, \mu_{a}:=\frac{\lambda}{k_{a}}$ and $r:=\frac{k_{e}}{k_{a}}=\frac{\mu_{a}}{\mu_{e}}$. These quantities are related to ones considered in [14].

For comparison with the random models below, we note the following facts:

- When $\mu_{a} \rightarrow 0$, we are in the case of instantaneous absorption, which is equivalent to the multi-IV case. In this frame, we recover the results of [11].

- In Formula (9), the term $G(r)$, given by Formula (10), is always negative, as may easily be checked.

- The term $G(r)$, seen as a function of $r$, is increasing. It is thus minimum when $r$ equals 0 , and it tends to 0 when $r$ tends to infinity.

- When $\mu_{e}$ tends to 0 , the variance tends to 0 at rate $\mu_{e}^{2}$ when $\mu_{a} \neq 0$ and at rate $\mu_{e}$ when $\mu_{a}=0$. 
- For a fixed mean $E_{d}=1$, and when $\mu_{e}$ tends to infinity, $\operatorname{Var}_{d}$ tends to 0 at speed $\frac{1}{\mu_{e}^{2}}$. When $\mu_{e}$ tend to 0 , the variance tends to $\frac{1}{2 \mu_{a}}$ when $\mu_{a} \neq 0$, and to infinity at speed $\frac{1}{\mu_{e}}$ when $\mu_{a}=0$.

- $\mathbb{P}\left(\left|C_{d}-\mathbb{E}\left(C_{d}\right)\right| \geq \gamma E\left(C_{d}\right)\right) \leq \frac{1}{\mu^{* 2} \gamma^{2}}$ for $\gamma$ large enough, where $\mu^{*}=\max \left\{\mu_{e}, \mu_{a}\right\}$ (see Formula (25) for a more precise expression). Assume $\mu_{a}, \mu_{e}$ tend to infinity at the same rate. Then, the bound is $\frac{1}{\mu^{* 4} \gamma^{2}}$.

These formulas quantify the obvious fact that the variability of concentration is a decreasing function of the number of takes per unit time. As we show below, non compliance amplifies the variability of the concentration in a way we will precisely measure.

\subsection{Full compliance with population parameter variability}

In the previous section, we have characterized the variability for a deterministic PK model defined by a unique set of PK parameters, i.e. the case of a single compliant subject with perfectly known pharmacokinetic parameters. We now investigate variability in a Pop-PK model for the case of a whole population. A Pop-PK involve randomly distributed PK parameter to reflect the peculiarity of each patient and other unexplained variability. A typical assumption is to consider that individual parameters are log-normally distributed in the population. The log-normal distribution is a common modelling for positive continuous quantities, and seems to fit well with the finding of large scale Pop-PK studies [8, 18]. We also assume that each subject in the population is fully compliant and that their drug intake frequency $\lambda$ is the same.

Let us first consider the case where the elimination rate is random and the other parameters such as $D, V_{d}, F, k_{a}$ are constants. This amounts to replacing $k_{e}$ by $k_{e} U_{k_{e}}$ in the preceding computations, where $k_{e}$ is the typical parameter value of the population and $U_{k_{e}}$ is a dimensionless random variable that represent the variability among subject, which is assumed to follows a log-normal distribution with parameters $m_{k_{e}}=0$ and $\sigma_{k_{e}}^{2}$. The choice $m_{k_{e}}=0$ ensures that the reference value $k_{e}$ is the median of the distribution. We wish to examine the impact of this variability on the long-term average concentration $E_{d}$, given by equations (6) and (8). The variability of $E_{d}$ is solely due to the variation of $k_{e} U_{k_{e}}$. In other words, we consider the variable $E_{d}\left(k_{e} U_{K_{e}}\right)$ with $U_{k_{e}}$ following a log-normal distribution, and measure its variability. Thus, the mean and variance of $E_{d}$ across the population are given by:

$$
\begin{gathered}
\mathbb{E}_{\text {pop }}\left(E_{d}\right)=\frac{\lambda}{k_{e}} \frac{F D}{V_{d}} \mathbb{E}_{\text {pop }}\left(\frac{1}{U_{k_{e}}}\right)=\frac{\lambda}{k_{e}} \frac{F D}{V_{d}} e^{\sigma_{k_{e}}^{2} / 2} \\
\operatorname{Var}_{p o p}\left(E_{d}\right)=\left(\frac{\lambda}{k_{e}} \frac{F D}{V_{d}}\right)^{2} \operatorname{Var}_{\text {pop }}\left(\frac{1}{U_{k_{e}}}\right)=\left(\frac{\lambda}{k_{e}} \frac{F D}{V_{d}}\right)^{2} e^{\sigma_{k_{e}}^{2}}\left(e^{\sigma_{k_{e}}^{2}}-1\right) .
\end{gathered}
$$

Let us rewrite these formulas in terms $\mu_{e}$ and the coefficient of variation of the elimi- 
nation rate $C V_{k_{e}}=\sqrt{e^{\sigma_{k_{e}}^{2}-1}}$ :

$$
\begin{gathered}
\mathbb{E}_{\text {pop }}\left(E_{d}\right)=\mu_{e} \frac{F D}{V_{d}} \sqrt{1+C V_{k_{e}}^{2}}, \\
\operatorname{Var}_{p o p}\left(E_{d}\right)=\left(\mu_{e} \frac{F D}{V_{d}}\right)^{2} C V_{k_{e}}^{2}\left(1+C V_{k_{e}}^{2}\right) .
\end{gathered}
$$

The same approach applies if one wishes to take into account in addition the variability of $V_{d}$ and $F$ across the population. In this case, $k_{a}$ is assumed constant and one simultaneously replaces $k_{e}$ by $k_{e} U_{k_{e}}, V_{d}$ by $V_{d} U_{V_{d}}$ and $F$ by $F U_{F}$, where $U_{k_{e}}, U_{V_{d}}$ and $U_{F}$ are random variables log-normally distributed with median parameters $m_{k_{e}}=0, m_{V_{d}}=0$, $m_{F}=0$ and variability parameters $\sigma_{k_{e}}^{2}, \sigma_{V_{d}}^{2}, \sigma_{F}^{2}$ respectively. To perform the computations, the knowledge of the joint distribution of $U_{k_{e}}$ and $U_{V_{d}}$, or at least that of $U_{k_{e}} U_{V_{d}}$ is needed. Joint distributions are not usually reported in Pop-PK studies, but since the product $k_{e} V_{d}$ is nothing but the clearance $C l$, we can see that in this case we have:

$$
C l=k_{e} U_{k_{e}} V_{d} U_{V_{d}}=C l_{0} U_{C l}
$$

where $C l_{0}=k_{e} V_{d}$ and $U_{C l}=U_{k_{e}} U_{V_{d}}$ represent the variability of clearance, whose distribution is more frequently reported in Pop-PK studies. Note that the log-normality of clearance typically reported in the literature is compatible with the joint log-normality of $\left(U_{k_{e}}, U_{V_{d}}\right)$. Thus, if we assume that $U_{C l}$ and $U_{F}$ are log-normally distributed random variables with parameters $m_{C l}=0, m_{F}=0$ and variability parameters $\sigma_{C l}^{2}, \sigma_{F}^{2}$ respectively, and we assume that the variables $U_{C l}$ and $U_{F}$ are correlated with correlation $\rho=\operatorname{cov}\left(\log \left(U_{k_{e}}\right), \log \left(U_{V_{d}}\right)\right) / \sigma_{C l} \sigma_{F} \in[-1,1]$, computations similar to the ones above lead to:

$$
\begin{gathered}
\mathbb{E}_{p o p}\left(E_{d}\right)=\mu_{e} \frac{F D}{V_{d}} \sqrt{1+C V^{2}}, \\
\operatorname{Var}_{p o p}\left(E_{d}\right)=\left(\mu_{e} \frac{F D}{V_{d}}\right)^{2} C V^{2}\left(1+C V^{2}\right),
\end{gathered}
$$

where $C V=\sqrt{e^{\sigma_{C l}^{2}+\sigma_{F}^{2}+2 \rho \sigma_{C l} \sigma_{F}-1}}$ is the coefficient of total variation. Note that individual variations of $F$ are difficult to distinguish from ones of $C l$. We have therefore chosen to control the variability through the coefficient of total variation $C V$, which encompasses the variability of $k_{e}, V_{d}$ and $F$.

For consistency, we will always use in the sequel the following PK parameters similar to those of imatinib, see $[2,3,19]$. They are given in Table 1 . 


\begin{tabular}{|c|c|c|c|c|}
\hline \hline Parameter & Unit & TV & SD & CV (\%) \\
& & & & \\
\hline$D$ & $m g$ & 150 & 0 & $0 \%$ \\
$k_{a}$ & $h^{-1}$ & 0.61 & 0 & $0 \%$ \\
$F$ & & 1 & 0.2462 & $25 \%$ \\
$C l$ & $l h^{-1}$ & 14.3 & 0.3491 & $36 \%$ \\
$V_{d}$ & $l$ & 347 & 0.5781 & $63 \%$ \\
\hline \hline
\end{tabular}

Table 1: PK parameters of imatinib. TV: Typical Value; SD: Standard Deviation; CV: Coefficient of variation.

Suppose that the correlation parameter $\rho$ is 0 . Then the coefficient of total variation is $C V=44.74 \%$, which correspond to a standard deviation of 0.4272 . Assuming that doses are taken every $\frac{1}{\lambda}=12 h$, one obtains for the mean and variance:

$$
\mathbb{E}_{\text {pop }}\left(E_{d}\right)=0.9576 \mathrm{mg} / \mathrm{l}, \quad \text { and } \quad \operatorname{Var}_{\text {pop }}\left(E_{d}\right)=0.1836 m g^{2} / l^{2},
$$

where we have assumed for simplicity that $V_{d}$ is constant, i.e. $C l=k_{e} V_{d} U_{k_{e}}$, where $U_{k_{e}}$ is log-normal with parameters $m_{k_{e}}=0$ and $\sigma_{k_{e}}^{2}=\sigma_{C l}^{2}$.

Note that the coefficient of total variation $C V$ is an increasing function of $\rho$, that reaches its maximum $C V_{\max }=65.22 \%$ when $\rho=1$ and its minimum $C V_{\min }=10.31 \%$ when $\rho=-1$.

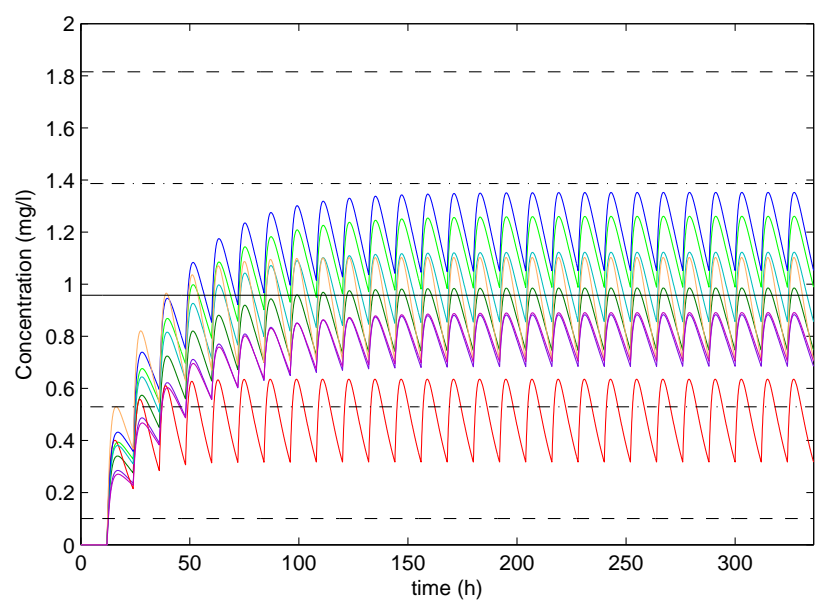

Figure 1: Eight sample paths of the concentration taking into account variability of clearance and bioavailability, graphed for the first fourteen days. The black solid line is the population mean $\mu_{p o p}$, the dotted-dashed lines correspond to the confidence bands $\mu_{p o p} \pm \sigma_{p o p}$ and the dashed lines to the confidence bands $\mu_{p o p} \pm 2 \sigma_{p o p}$.

We display on Figure 1 eight sample evolutions of the concentration for the first 14 days in this scenario. The simulations show that a steady state is quickly reached. In addition, all 
but one samples lie within one standard deviation $\sigma_{p o p}=\sqrt{\operatorname{Var}_{p o p}\left(E_{d}\right)}$ of the population mean $\mu_{\text {pop }}=\mathbb{E}_{\text {pop }}\left(E_{d}\right)$.

In the following sections, we return to the case of a single subject with perfectly known PK parameters, but with poorly compliant behaviour, and we will compare the variability in the various cases.

\subsection{Continuous model with random intake instants and deter- ministic doses}

In the context of poor compliance the $t_{i}$ are not fixed, but are rather modelled as random variables. We denote these stochastic time instants as $\left(T_{i}\right)_{\in \mathbb{N}}$ and we assume that the intervals $S_{i}=T_{i}-T_{i-1}$ between two doses are i.i.d. with exponential distribution of parameter $\lambda$. In other words, the sequence $\left(T_{i}\right)_{i \in \mathbb{N}}$ is supposed to be a homogeneous Poisson process, and the mean duration between two drug intakes is equal to $\frac{1}{\lambda}$. The stochastic concentration at time $t$ reads :

$$
C(t)=\frac{F D}{V_{d}} \frac{k_{a}}{k_{a}-k_{e}} \sum_{i}\left(e^{-k_{e}\left(t-T_{i}\right)}-e^{-k_{a}\left(t-T_{i}\right)}\right) \mathbb{1}_{\left(t \geq T_{i}\right)}
$$

We illustrate this model on Figure 2, where we display eight sample paths of the evolution of $C(t)$, simulated using the parameters given in Table 1 and $\lambda^{-1}=12 h$.

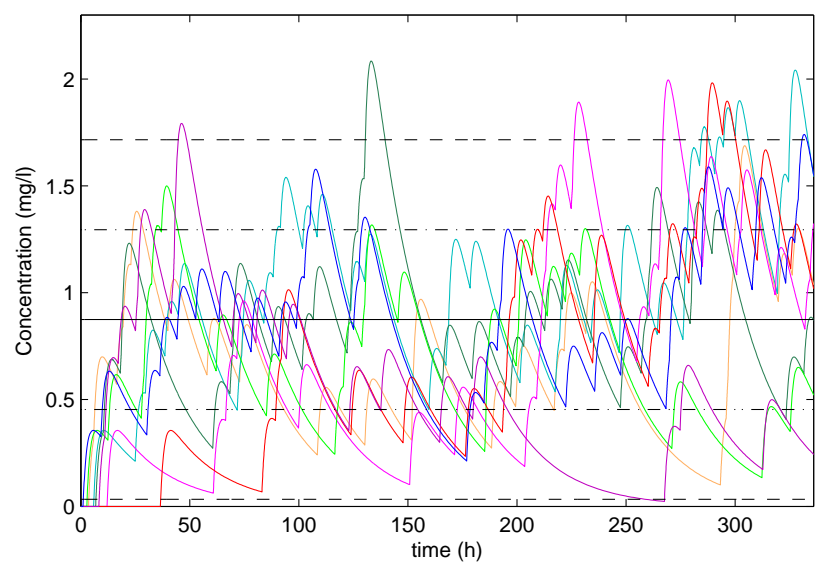

Figure 2: Eight sample paths of the concentration with Poisson distributed instant of intakes, for the first 14 days The black solid line is the limit concentration mean $\mathbb{E}(C)$, the dotted-dashed lines delineate the confidence bands $\mathbb{E}(C) \pm \sqrt{\operatorname{Var}(C)}$ and the dashed lines to the confidence bands $\mathbb{E}(C) \pm 2 \sqrt{\operatorname{Var}(C)}$.

We will show in Section 3 that $C(t)$ has a well defined limit when $t$ tends to infinity. We are interested in the variability and regularity properties of this steady state, or asymptotic 
concentration, which is denoted $C$. The following equations are established in Section 3.1:

$$
\begin{gathered}
\mathbb{E}(C)=\mu_{e} \frac{F D}{V_{d}}, \\
\operatorname{Var}(C)=\frac{\mu_{e}}{2}\left(\frac{F D}{V_{d}}\right)^{2} \frac{1}{1+r} .
\end{gathered}
$$

In view of (14) and (15), we remark that:

- As expected, the means are the same in the deterministic and random models.

- As is intuitively obvious, the variance in the case of full compliance is always smaller than the one in the random situation. This can be observed from the fact that the term $G(r)=\frac{2}{1-r^{2}}\left(\frac{1}{e^{1 / \mu_{e}}-1}-\frac{r}{e^{1 / r \mu_{e}}-1}\right)-2 \mu_{e}$ in Formula (9) is always negative. Formulas (9) and (15) quantify in a precise way how much variability is increased as an effect of poor compliance.

- When $\mu_{e}$ tends to 0 , the variance tend to 0 , with the same speed as in the deterministic model.

- For a fixed mean equal to 1 , when $\mu_{e}$ tends to infinity, the variance tends to 0 at speed $\frac{1}{\mu_{e}}$. This is to be compared to the faster speed of convergence of $\frac{1}{\mu_{e}^{2}}$ in the deterministic case. When $\mu_{e}$ tend to 0 , the variance tends to $\frac{1}{2 \mu_{a}}$ for $\mu_{a} \neq 0$, which is strictly larger than the corresponding limit in the deterministic case. Finally, when $\mu_{a}=0$, the variance tends to infinity at same rate as in the deterministic model $\left(\frac{1}{\mu_{e}}\right)$.

- $\mathbb{P}(|C-\mathbb{E}(C)| \geq \gamma E(C)) \leq \frac{1}{2 \mu^{*} \gamma^{2}}$ for $\gamma$ enough large and for all $\mu^{*}$ (recall that $\left.\mu^{*}=\max \left\{\mu_{e}, \mu_{a}\right\}\right)$. This is a slower speed of convergence than in the deterministic case, which is $\frac{1}{\mu^{* 2}}$.

- The probability that the long-term concentration exceeds a given level $\gamma$ decays as $\frac{1}{\gamma^{2}}$. More precisely, $\mathbb{P}(C \geq \gamma) \leq \frac{7}{6 \gamma^{2}}\left(\frac{F D}{V_{d}}\right)^{2} \mu_{e}^{2}\left(1+\frac{1}{2\left(\mu_{e}-\mu_{a}\right)}\right)$. If $\mathbb{E}(C)=\mu_{e} \frac{F D}{V_{d}}$ is assumed to be constant, one sees that this probability decays at the same speed for all values of $\mu_{e}, \mu_{a}$.

The coefficient of variation of the limit concentration $C$ is $1 / \sqrt{2\left(\mu_{e}+\mu_{a}\right)}$; as a consequence, variability is larger for smaller values of $\mu^{*}$, i.e. when the absorption process or the elimination process is fast compared to the mean frequency of drug intake. In Section 3.2, we will show that $\mu^{*}$ also controls the regularity of the limit concentration distribution.

The difference between the variance $\operatorname{Var}_{d}$ and $\operatorname{Var}(C)$, given by equations (9) and (15) respectively, is largest when $r$ is small, and vanishes when $r$ tends to infinity. The interpretation of this fact is straightforward: for a fixed $k_{e}$, a large $r$ means a small $k_{a}$ and thus a slow absorption. In this case, the effect of a drug intake on the system takes a very long time to appear, and thus a random delay in the intake has almost no effect. On the contrary, a small $r$ translates into fast absorption, and any irregularity in the schedule has 
a noticeable impact. We also remark that, because of the "damping" introduced by $k_{a}$ in the oral model, the increase of variance due to random intakes is smaller here than in the intravenous model studied in [11]. When $r$ tends to 0 , we recover the results of [11] since the damping vanishes. Again, the formulas above quantify precisely these effects.

In a way similar to what was observed in the deterministic model, the variability of concentration is a decreasing function of the expected number of takes per unit time $\lambda^{-1}$ : as is intuitively clear, increasing the mean frequency of intakes while keeping constant the average quantity of administrated drug diminishes the negative impact of poor compliance in terms of the probability of departing significantly from the mean concentration. In order to compare the variances in the deterministic (9) and non compliant cases (15), we plot their evolutions as a function of $\mu_{e}$ and $\mu_{a}$ in two situations. First, in Figure 3, we let $\mu_{e}$ and $\mu_{a}$ vary and keep the other parameters, i.e. $D, F$ and $V d$, constants. This corresponds for instance to the case where the number of doses per unit time (or average number of doses per unit time in the stochastic case) evolves, while maintaining everything else unchanged.

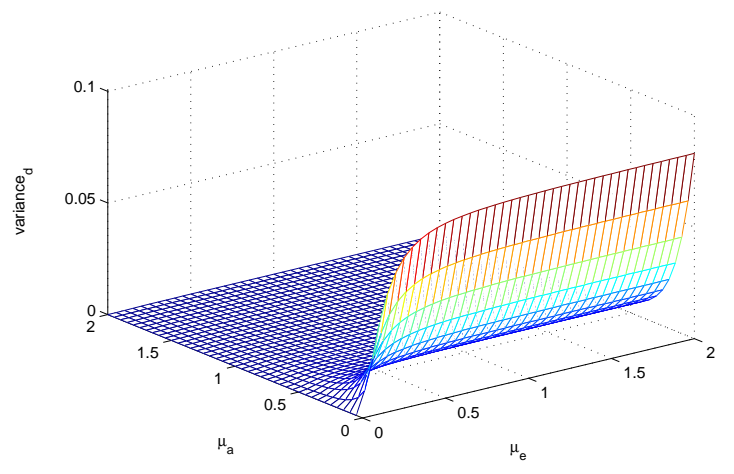

(a) Deterministic case

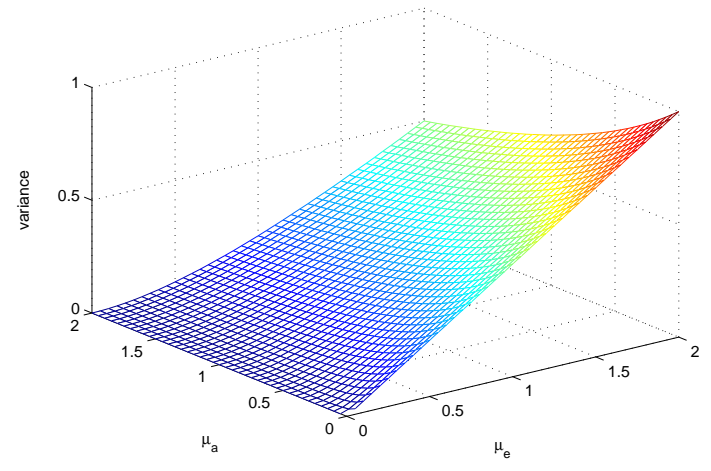

(b) Random case

Figure 3: Evolution of the variance as a function of $\mu_{e}$ and $\mu_{a}$, when all other parameters are kept constant.

As can be seen on Figure 3, when $\mu_{e}$ tends to infinity, the variance in the deterministic case reaches a plateau, while the random situation leads to an unbounded variance. Hence non-compliance has here a dramatic effect. When $\mu_{e}$ tends to 0 , both variances tend to 0 , at rate $\mu_{e}^{2}$ when $\mu_{a} \neq 0$ and at rate $\mu_{e}$ when $\mu_{a}=0$.

The conclusions in this frame are however somewhat unrealistic, since they lead to an unbounded increase of the mean when $\mu_{e}$ tends to infinity. This is why, in Figure 4, we consider the more reasonable situation where $\mu_{e}$ and $\mu_{a}$ vary but the mean is kept constant. From equations (9) and (15), one sees that this simply translates into ensuring that $\mu_{e} \frac{F D}{V d}$ is constant. Thus we are in the case where, for instance, one increases the frequency of drug intake while decreasing accordingly the unit dose. Here, we take $\mu_{e} \frac{F D}{V d}=0.8743$, which corresponds to the parameters given in Table 1 , and $\lambda^{-1}=12 h$. Figure 4 indicates that 
both variances tend to 0 when $\mu_{e}$ tends to infinity. The speed of convergence is however faster, as expected, in the deterministic case $\left(\frac{1}{\mu_{e}^{2}}\right)$ than in the random one $\left(\frac{1}{\mu_{e}}\right)$. Consider now the case where $\mu_{e}$ tend to 0 : when $\mu_{a} \neq 0$, the variance in the random frame tends to $\frac{1}{2 \mu_{a}}$ and to a strictly smaller value in the deterministic case. When $\mu_{a}=0$, both variances tend to infinity at same rate $\left(\frac{1}{\mu_{e}}\right)$.

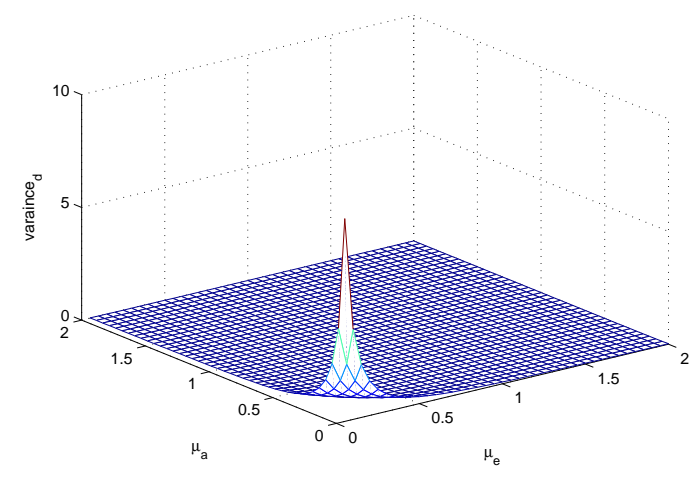

(a) Deterministic case

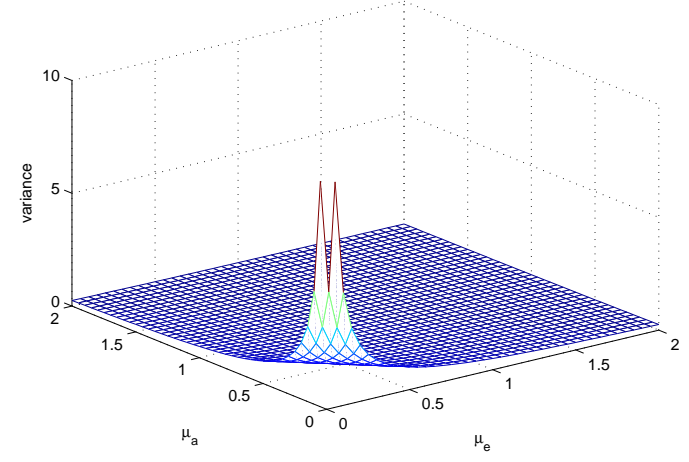

(b) Random case

Figure 4: Evolution of the variance as a function of $\mu_{e}$ and $\mu_{a}$ when mean is kept constant.

An interesting comparison is to plot the values of $\lambda$ in the random model as a function of the ones of $\lambda$ in the deterministic one that yield the same variance, as shown in Figure 5.

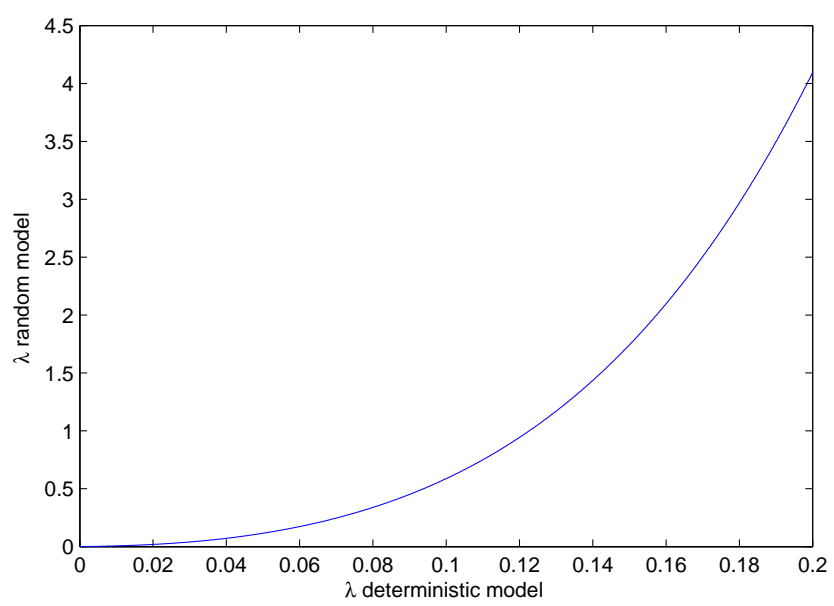

Figure 5: Average number of doses per hour in the non-compliant case as a function of the number of doses per hour in the fully compliant one yielding same variance for the concentration. 
For instance, the point $(1 / 24 ; 0.079)$ in the graph on Figure 5 means that a compliant patient taking a dose every day will have same concentration variability as a non compliant one taking in average a dose every $12.66=1 / 0.079 h$, when the mean concentration, mean dose per day and all other parameters are the same in both situations.

Let us now compare the variabilities induced by non compliance in the random instants model and by differing PK-parameters in a fully compliant population as studied in Section 2.2. We consider the case where $k_{a}$ and $V_{d}$ are constant and $k_{e}, F$ are replaced by $k_{e} U_{k_{e}}$ and $F U_{F}$, with $U_{k_{e}}$ and $U_{F}$ log-normally distributed random variables with median parameters $m_{k_{e}}=0, m_{F}=0$ and variability parameters $\sigma_{k_{e}}^{2}, \sigma_{F}^{2}$ respectively. As in Figure 5, we plot on Figure 6 the values of $\lambda$ in the random model as a function of $\sigma_{k_{e}}^{2}$ and $\sigma_{F}^{2}$ that yield the same variance of concentration when the mean of concentration, the mean dose per day and the other parameters are the same in both situations. From equations (11), (12), (14) and (15), one sees that this amounts to setting:

$$
\lambda=\frac{k_{e}}{2(1+r)\left(e^{\sigma_{k_{e}}^{2}+\sigma_{F}^{2}}-1\right)},
$$

where $\lambda$ is the mean number of doses per hours of the non compliant patient, $k_{e}$ is both his elimination rate and the mean elimination rate in the population, $\sigma_{k_{e}}^{2}$ and $\sigma_{F}^{2}$ are the variances of the elimination rate and the bioavailability in the population respectively.

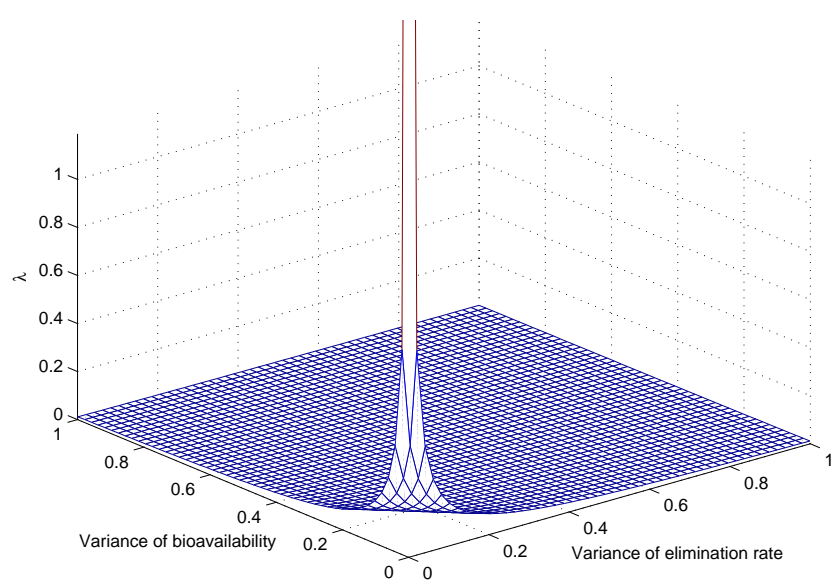

Figure 6: Average number of doses per hour in the non-compliant case as a function of the variance of the elimination rate and variance of bioavailability, in a fully compliant population yielding same variance for the concentration.

For example, the point $(0.12,0.06,0.1)$ in the graph on Figure 6 has the following meaning: the variance of the concentration for a population of compliant patients with $\sigma_{k_{e}}^{2}=0.12$ and $\sigma_{F}^{2}=0.06$ is the same as the one of a single non compliant patient taking in average a dose every $10(=1 / 0.1)$ hours. Likewise, a single non compliant patient taking 
in average a dose every day displays the same variance in concentration as a population of fully compliant individuals with $\sigma_{k_{e}}^{2}+\sigma_{F}^{2}=0.38$, i.e. a coefficient of total variation equal to $68 \%$. Note that the same conclusions hold if one consider a population where the clearance instead of the elimination rate follows a lognormal distribution.

Let us now consider the regularity of the distribution of $C$. It is shown in Section 3.2 that the smoothness of the distribution of the concentration is governed by $\mu^{*}=\max \left\{\mu_{e}, \mu_{a}\right\}$. We prove that, in the long term, the cumulative probability distribution of drug concentration may display two types of behaviours: when $\mu^{*}$ is larger than one, the distribution is regular, while, when it is smaller than one, it is singular only at the origin. Thus, $\mu^{*}=1$ is the critical value below which the moments of intakes are too scarce with respect to the elimination and absorption rates, resulting in a high probability of having too small a concentration of drugs.

To illustrate this fact, we plot on Figure 7 histograms representing the empirical probability distribution of $C(T)$, for a fixed time $T$, in two particular cases: $\mu^{*}=2$ and $\mu^{*}=0.5$. The value of $D$ in each case was adjusted accordingly in order to keep a constant mean. These histograms were obtained by simulating 50, 000 independent sample paths of concentration in each scenario until time $T$ and distributing the outcomes into 100 evenly spaced bins. The time $T$ was chosen large enough so that the steady state has been reached (we have set $T=100 \max \left\{1 / \lambda, 1 / k_{e}\right\}$ ). The singularity of the distribution of $C$ at the origin in the case $\mu^{*}<1$ (second histogram) manifests itself through the sharp spike in the first bin.

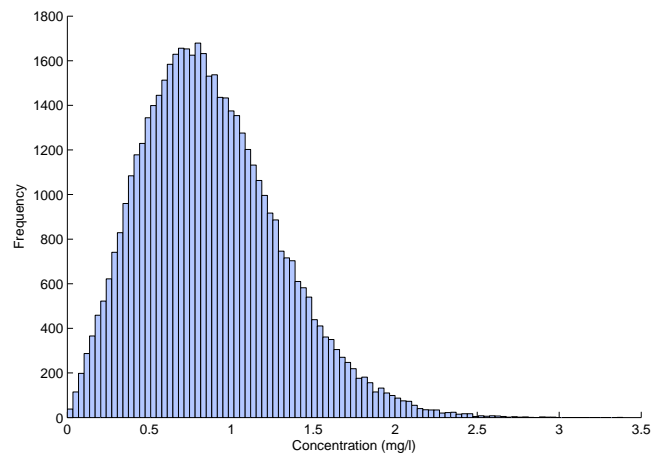

(a) $\mu^{*}=2$

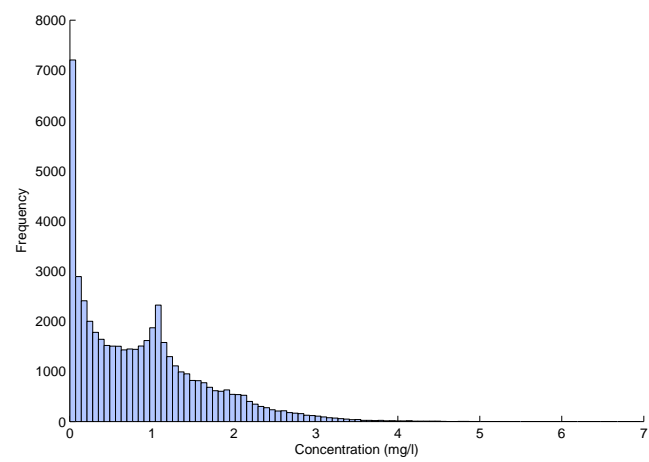

(b) $\mu^{*}=0.5$

Figure 7: Histogram of the distribution of $\mathrm{C}$

The reader is referred to Section 3 for more details on this model. 


\subsection{Continuous model with random intake instants and random doses}

In this section, we sum up the results obtained in Section 4, where we generalize the continuous model of Section 2.3 to allow for random doses. The idea is that the careless patient that has an irregular schedule is likely to also mess with the doses. For instance, he might take a double dose to make up for a missing one. Formally, this translates into the fact that the quantity $D$ in (5) is now a random variable that may vary at each take rather than being a constant. In other words, the concentration, denoted $C^{r d}(t)$, is now given by:

$$
C^{r d}(t)=\frac{F D}{V_{d}} \frac{k_{a}}{k_{a}-k_{e}} \sum_{i} D_{i}\left(e^{-k_{e}\left(t-T_{i}\right)}-e^{-k_{a}\left(t-T_{i}\right)}\right) \mathbb{1}_{\left(t \geq T_{i}\right)},
$$

where $D, F$ and $V_{d}$ are constants, the $\left(T_{i}\right)_{i \in \mathbb{N}}$ again form a homogeneous Poisson process, and the $\left(D_{i}\right)_{i \in \mathbb{N}}$ are random variables. The dose taken at time $T_{i}$ is $D D_{i}$. It thus seems natural to assume that $\mathbb{E}\left(D_{i}\right)=1$ (i.e on average, the patient takes the required dose), that $D_{i}$ is supported on $\mathbb{R}_{+}$, and that it has compact support, i.e. the patient cannot take arbitrarily large doses (although we shall need only weaker assumptions).

We illustrate this model by showing on Figures 8 and 9 simulated sample paths of the concentration in two particular cases considered in more details in Section 4: in the first one, the random factors $D_{i}$ follow a uniform distribution on an interval $[a, b]$, while in the second one they follow a discrete distribution taking two possible values $d_{1}$ and $d_{2}$ with probability $q_{1}, q_{2}$.

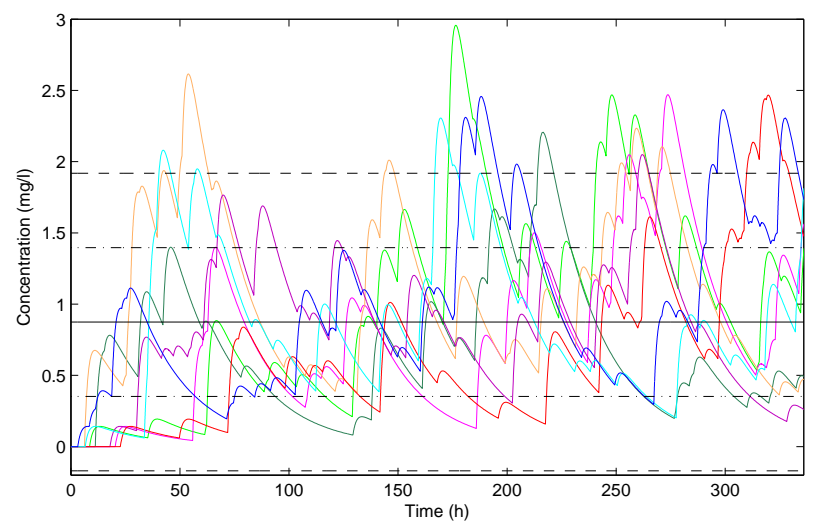

Figure 8: Eight sample paths of the concentration with Poisson distributed instant of intakes and discretely distributed random doses, for the first 14 days. The black solid line is the limit concentration mean $E\left(C^{r d}\right)$, the dotted-dashed lines correspond to the confidence bands $E\left(C^{r d}\right) \pm \sqrt{\operatorname{Var}\left(C^{r d}\right)}$ and the dashed lines to the confidence bands $E\left(C^{r d}\right) \pm 2 \sqrt{\operatorname{Var}\left(C^{r d}\right)}$.

The PK parameters chosen for the simulation of the sample paths are the ones given in Table 1 with an average time between intakes equal to $\lambda^{-1}=12 h$. The parameters for the random doses in both cases are given in Table 2. 


\begin{tabular}{l|l|l|l|l|l|}
\hline \hline$d_{1}=0.4$ & $d_{2}=1.9$ & $q_{1}=0.6$ & $q_{2}=0.4$ & $a=0.2$ & $b=1.8$ \\
\hline \hline
\end{tabular}

Table 2: Numerical values of the parameters of the random doses distributions

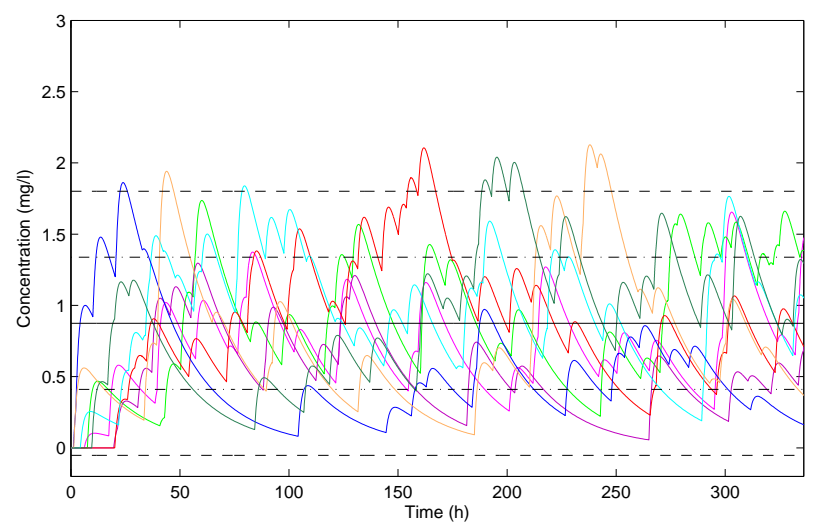

Figure 9: Eight sample paths of the concentration with Poisson distributed instant of intakes and uniformly distributed random doses, for the first 14 days. The black solid line is the limit concentration mean $E\left(C^{r d}\right)$, the dotted-dashed lines correspond to the confidence bands $E\left(C^{r d}\right) \pm \sqrt{\operatorname{Var}\left(C^{r d}\right)}$ and the dashed lines to the confidence bands $E\left(C^{r d}\right) \pm 2 \sqrt{\operatorname{Var}\left(C^{r d}\right)}$.

We prove in Section 4.1 that the mean value in this model is the same as in the previous ones, i.e.

$$
\mathbb{E}\left(C^{r d}(t)\right)=\mathbb{E}(C(t)) .
$$

Furthermore, under the assumption that the doses $\left(D_{i}\right)_{i \in \mathbb{N}}$ do no depend on the times $\left(T_{i}\right)_{i \in \mathbb{N}}$, we show the following equality:

$$
\operatorname{Var}\left(C^{r d}(t)\right)=\left(1+\operatorname{Var}\left(D_{1}\right)\right) \operatorname{Var}(C(t)) .
$$

In other words, the variance in the case of random dosing is simply the one of the deterministic dose case multiplied by $1+\operatorname{Var}\left(D_{1}\right)$. As a consequence, estimating quantities such as $\mathbb{P}(|C-\mathbb{E}(C)| \geq \gamma \mathbb{E}(C))$ or $\mathbb{P}(C \geq \gamma)$ or comparing to the case of (population) full compliance is readily done once $\operatorname{Var}\left(D_{1}\right)$ is known. In Section 4.1, we detail two particular cases: the first one is where $D_{i}$ is uniformly distributed on a interval, and the second one is where it takes values in a discrete set. We characterize the situations leading to the largest variances for both distributions (formulas (33) and (35)).

In contrast to variability, random dosing does not affect the regularity of the distribution of the long term concentration: this distribution is again smooth when $\mu^{*}>1$ and is singular at 0 otherwise. 


\subsection{Discrete model with random intake instants and determin- istic doses}

We study a time-discretized version of the random model presented in Section 2.3. The general idea is that, instead of taking the drug at arbitrary time instants $t \in \mathbb{R}^{+}$, the patient will only do so at times which form a random subset of $\left\{t_{l}^{n}=l / n, l \in \mathbb{N}\right\}$, where $n$ is a fixed number. There are two main reasons for considering such a model. First, there are indeed natural situations where a time discretization does occur. For instance, the medication must sometimes be taken at precise moments, like before lunch. Many people will always have their lunch at a fixed time, like certain workers or many older people. Second, as we show in Section 5.3, the time discretized model tends to the continuous one when the discretization step tends to 0 . Thus, for $n$ large enough, the practical difference between both models vanishes. Nonetheless, the discrete model displays various interesting and intriguing features that are not present in the continuous one. Let us finally mention that considering the discretized model is very close to sampling the concentration of the continuous one. Since blood concentrations cannot be monitored continuously, the outcome of any clinical study is discrete in nature, which gives further justification for the discrete model.

We show in Section 5.1 that the steady state discretized concentration $Y_{(p)}$ in the situation where the patient takes a dose $D$ with probability $p$ independently at each time $j / n$ reads:

$$
Y_{(p)}=\frac{F D}{V_{d}} \frac{k_{a}}{k_{a}-k_{e}} \sum_{j=0}^{\infty}\left(\beta_{e}^{j}-\beta_{a}{ }^{j}\right) X_{j},
$$

where the $\left(X_{j}\right)_{j \in \mathbb{N}}$ are i.i.d. Bernoulli random variables with parameter $p$ (i.e. $X_{j}=0$ with probability $1-p$ and $X_{j}=1$ with probability $p$ ). The parameter $\beta_{e}=e^{-k_{e} / n}$ is the elimination rate for one time step while $\beta_{a}=e^{-k_{a} / n}$ is the absorption rate for one time step. As explained in Section 5.1, in the discrete model, the number of drug intakes per unit time is $n p$, thus one has to set $p=\frac{\lambda}{n}$ to ensure correspondence with the continuous model.

Set $\alpha:=\frac{F D}{V_{d}} \frac{k_{a}}{k_{a}-k_{e}}$. The mean and variance of the discretized concentration are given by the following formulas:

$$
\begin{aligned}
E_{\text {disc }} & :=\mathbb{E}\left[Y_{(p)}\right]=\alpha p\left(\frac{1}{1-\beta_{e}}-\frac{1}{1-\beta_{a}}\right), \\
\operatorname{Var}_{\text {disc }} & :=\operatorname{Var}\left(Y_{(p)}\right)=\alpha^{2} p(1-p)\left(\frac{1}{1-\beta_{e}^{2}}-\frac{2}{1-\beta_{e} \beta_{a}}+\frac{1}{1-\beta_{a}^{2}}\right) .
\end{aligned}
$$

Note that $E_{d i s c}$ and $V a r_{d i s c}$ tend respectively to the mean and variance in the continuous model with random instants of intakes when $p$ tends to 0 .

If ones fixes $E_{d i s c}=1$, then the variance in the discrete model becomes:

$$
\operatorname{Var}_{d i s c}=\frac{1-p}{p} \frac{\left(1-\beta_{e}\right)\left(1-\beta_{a}\right)\left(1+\beta_{e} \beta_{a}\right)}{\left(1+\beta_{e}\right)\left(1+\beta_{a}\right)\left(1-\beta_{e} \beta_{a}\right)} .
$$


Thus $\operatorname{Var}_{d i s c}$ tends to 0 at speed $\frac{1-p}{2 \mu_{e}}$ when $\mu_{e}$ tends to infinity, which is the same rate as $V a r$, the variance in the continuous random model of Section 3.1. When $\mu_{e}$ tends to 0, $\operatorname{Var}_{\text {disc }}$ tends to $\frac{1-p}{p} \frac{1-\beta_{a}}{1+\beta_{a}}$ in contrast to both the deterministic and random continuous cases, where the variance tends to infinity. Figure 10 displays the evolution of the ratio $V a r_{d i s c} / V a r$ as a function of $\mu_{e}$ and $\mu_{a}$ when the mean is kept constant.

Formula (36) in Section 5.2 gives the probability that the concentration departs significantly from its mean. It is interesting to compare it with (24) to contrast the impact of non-compliance in the discrete and continuous time models, and to (25) to measure the added variability with respect to the fully compliant situation.

The study of the regularity of the distribution is much more involved in the discrete case than in the continuous one, as the former may display a fractal behaviour. Indeed, for some values of the parameters, the distribution will be everywhere singular. This means that the evolution of the distribution varies wildly for most values of the concentration. In addition, the dichotomy smooth/singular is not governed by $\mu^{*}$ alone, but by complex relations between $k_{e}, k_{a}$ and the discretization step. More precisely, the relevant parameter here is $\beta^{*}=\beta_{e} \vee \beta_{a}$ and the regularity of the discretized model is very different depending on whether $\beta^{*}<\frac{1}{2}$ or $\beta^{*} \geq \frac{1}{2}$.

When $\beta^{*}<\frac{1}{2}$, the distribution of $Y_{(p)}$ is singular with respect to the Lebesgue measure. This means that, in this situation, the probability distribution of the concentration will be highly irregular : it will vary erratically, taking only very particular values, and the probability that it ranges in some interval varies wildly when the interval changes. Note that $\beta^{*}<\frac{1}{2}$ is equivalent to $p>\mu^{*} \log (2)$ or $k^{*}>n \log (2)$ with $k^{*}=k_{e} \wedge k_{a}$. This reflects the fact that, for fixed $k^{*}$, the time instants at which the patient is supposed to take his drugs are sufficiently spaced, and that he forgets to do so randomly at some of these instants.

The case $\beta^{*}>\frac{1}{2}$ is mathematically much more delicate and is not completely settled. What is known is that the distribution of $Y_{(p)}$ is absolutely continuous for almost all $\beta^{*}$ in $\left(\frac{1}{2}, 1\right)$ when $p=\frac{1}{2}$ : in effect, this means that the distribution of the concentration has a probability density and thus we are in the usual situation where the probability that the concentration ranges in some interval varies smoothly when the interval changes.

See Section 5.4 for more details.

\subsection{Discrete model with random intake instants and random doses}

Similarly to Section 2.4 , we consider a random dose variant of the previous model. The steady state concentration now reads:

$$
Y_{(p)}^{r d}=\alpha \sum_{j=0}^{\infty}\left(\beta_{e}^{j}-\beta_{a}^{j}\right) D_{j} X_{j},
$$

which accounts for the fact that the stochastic dose at time $j / n$ is equal to 0 or $D D_{j}$, where the random variables $D_{j}$ are i.i.d. with mean 1 and take values in an interval $\left[d_{m}, d_{M}\right]$ with 
$0<d_{m}<d_{M}<\infty$. The mean $E_{d i s c}^{r d}$ and variance $\operatorname{Var}_{\text {disc }}^{r d}$ in the random-dose discretized model are given by (see Section 6):

$$
\begin{aligned}
E_{d i s c}^{r d} & =\alpha p\left(\frac{1}{1-\beta_{e}}-\frac{1}{1-\beta_{a}}\right)=E_{d i s c}, \\
V a r_{d i s c}^{r d} & =\left(\frac{\operatorname{Var}\left(D_{1}\right)}{1-p}+1\right) \operatorname{Var}_{d i s c} .
\end{aligned}
$$

Random-dosing thus results in multiplying the variance of the deterministic-dose discretized model by $\frac{\operatorname{Var}\left(D_{1}\right)}{1-p}+1$. It is then easy to study the variability of the random-dose discretized model using the analysis developed in Section 5.2 for the discretized model.

We do not have at this time any significant result concerning the regularity of the distribution of the concentration in this model.

This ends the overview of our models and their main features. The reader will find in the next sections the precise derivations of the results mentioned above, along with a more detailed analysis of the models.

\section{Continuous model with random intake instants and deterministic doses}

In this section we study the main properties of the random concentration given by (13), which we recall here for convenience:

$$
C(t)=\frac{F D}{V_{d}} \frac{k_{a}}{k_{a}-k_{e}} \sum_{i}\left(e^{-k_{e}\left(t-T_{i}\right)}-e^{-k_{a}\left(t-T_{i}\right)}\right) \mathbb{1}_{\left(t \geq T_{i}\right)}
$$

where $\left(T_{i}\right)_{i \in \mathbb{N}}$ is a homogeneous Poisson process with parameter $\lambda$. The probability distribution of $C(t)$ may be described through its characteristic function $\varphi_{t}$. It is easily obtained by applying Campbell's theorem [9]. In our case, this yields:

$$
\begin{aligned}
\varphi_{t}(\theta)= & \exp \left\{\lambda \int_{0}^{t}\left(\exp \left\{i \theta \alpha\left(e^{-k_{e}(t-x)}-e^{-k_{a}(t-x)}\right)\right\}-1\right) d x\right\} \\
& \times \exp \left\{i \theta \alpha\left(e^{-k_{e} t}-e^{-k_{a} t}\right)\right\} .
\end{aligned}
$$

where we recall that $\alpha:=\frac{F D}{V_{d}} \frac{k_{a}}{k_{a}-k_{e}}$.

The change of variable $u=e^{-(t-x)}$ leads to:

Proposition 1. The characteristic function of $C(t)$ is

$$
\varphi_{t}(\theta)=\exp \left\{\lambda \int_{e^{-t}}^{1} \frac{\exp \left\{i \theta \alpha\left(u^{k_{e}}-u^{k_{a}}\right)\right\}-1}{u} d u+i \theta \alpha\left(e^{-k_{e} t}-e^{-k_{a} t}\right)\right\} .
$$




\subsection{Variability of the concentration}

The characteristic function allows us to compute the mean and variance of $C(t)$ :

$$
\mathbb{E}(C(t))=\lambda \alpha\left(\frac{1-e^{-k_{e} t}}{k_{e}}-\frac{1-e^{-k_{a} t}}{k_{a}}\right)+\alpha\left(e^{-k_{e} t}-e^{-k_{a} t}\right),
$$

so that, in the long term,

$$
\lim _{t \rightarrow \infty} \mathbb{E}[C(t)]=\mu_{e} \frac{F D}{V_{d}},
$$

Likewise, the variance of $C(t)$ reads:

$$
\operatorname{Var}(C(t))=\lambda \alpha^{2}\left(\frac{1-e^{-2 k_{e} t}}{2 k_{e}}+\frac{1-e^{-2 k_{a} t}}{2 k_{a}}-2 \frac{1-e^{-\left(k_{a}+k_{e}\right) t}}{k_{a}+k_{e}}\right),
$$

from which one gets

$$
\operatorname{Var}=\lim _{t \rightarrow \infty} \operatorname{Var}(C(t))=\frac{\mu_{e}}{2} \frac{1}{1+r}\left(\frac{F D}{V_{d}}\right)^{2},
$$

with $r:=\frac{k_{e}}{k_{a}}$.

Note that the convergence in (20) and (21) are exponential: only a few cycles are needed before the steady state is reached. The same remark applies to all convergences below.

In the next section, we show that, when $t$ tends to infinity, $C(t)$ also converges to a well defined random variable, denoted $C$, and we investigate in details some of its properties. Before, let us give a final result of interest pertaining to the variability of the concentration in the non-compliant case. More precisely, the following proposition yields bounds on the probability that the concentration exceeds a given (large) level, or departs significantly from its mean.

Proposition 2. For $\gamma$ large enough,

$$
\begin{gathered}
\mathbb{P}(C(t) \geq \gamma) \leq \frac{7}{6 \gamma^{2}}\left(\frac{F D}{V_{d}}\right)^{2}\left(\frac{\mu_{e}}{\mu_{e}-\mu_{a}}\right)^{2} H\left(t, k_{e}, k_{a}, \mu_{e}, \mu_{a}\right), \\
\mathbb{P}(C \geq \gamma) \leq \frac{7}{6 \gamma^{2}}\left(\frac{F D}{V_{d}}\right)^{2} \mu_{e}^{2}\left(1+\frac{1}{2\left(\mu_{e}-\mu_{a}\right)}\right) . \\
\mathbb{P}(|C-\mathbb{E}[C]| \geq \gamma E[C]) \leq \frac{1}{2 \mu_{e} \gamma^{2}} \frac{1}{1+r} .
\end{gathered}
$$

where

$$
\begin{aligned}
H\left(t, k_{e}, k_{a}, \mu_{e}, \mu_{a}\right)= & {\left[\left(\mu_{e}-1\right)\left(1-e^{-k_{e} t}\right)-\left(\mu_{a}-1\right)\left(1-e^{-k_{a} t}\right)\right]^{2} } \\
& +\left[\frac{\mu_{e}}{2}\left(1-e^{-2 k_{e} t}\right)-\frac{\mu_{a}}{2}\left(1-e^{-2 k_{a} t}\right)\right] .
\end{aligned}
$$


Proof. This is a direct application of the classical bound (see, e.g. [12], p. 209):

$$
\mathbb{P}(C(t) \geq \gamma) \leq 7 \gamma \int_{0}^{\frac{1}{\gamma}}\left(1-\operatorname{Re}\left(\varphi_{t}(\theta)\right)\right) d \theta
$$

valid for $\gamma>0$, and where $R e$ denotes the real part. Indeed,

$$
\begin{aligned}
\operatorname{Re}\left(\varphi_{t}(\theta)\right)= & \cos \left(\theta \alpha\left(e^{-k_{e} t}-e^{-k_{a} t}\right)+\lambda \int_{e^{-t}}^{1} \frac{\sin \left(i \theta \alpha\left(u^{k_{e}}-u^{k_{a}}\right)\right)}{u} d u\right) \\
& \times \exp \left\{\lambda \int_{e^{-t}}^{1} \frac{\cos \left(\theta \alpha\left(u^{k_{e}}-u^{k_{a}}\right)\right)-1}{u} d u\right\}
\end{aligned}
$$

and routine estimates yield (22). Inequality (23) follows in a similar way. Finally, (24) is simply Chebychev inequality.

Note that, in the deterministic case (full compliance), and with the same definition of the variance given by (7) in Section 2.1, one has in place of (24):

$$
\mathbb{P}\left(\left|C_{d}(t)-\mathbb{E}\left[C_{d}\right]\right| \geq \gamma E\left[C_{d}\right]\right) \leq \frac{1}{2 \mu_{e} \gamma^{2}}\left[\frac{1}{1+r}+G(r)\right],
$$

with $G(r)$ given by (10). This is another quantitative way to measure by how much the probability of differing from the mean will be larger in the non-compliant case. For instance, when $\mu_{e}$ tends to infinity but $\mu_{a}$ remains bounded (which implies that $r$ tends to 0 ), the above bound is of the order of $\frac{1}{\mu_{e}^{2} \gamma^{2}}$, and thus much smaller than the one in (24). When $\mu_{a}$ tends to infinity but $\mu_{e}$ remains bounded (i.e. $r$ tends to infinity), the bound is of the order of $\frac{1}{\mu_{a}^{2} \gamma^{2}}$, again much smaller than the one in (24). Finally, when $\mu_{e}$ and $\mu_{a}$ tend to infinity at the same rate, the bound in the deterministic case is of the order of $\frac{1}{\mu_{e}^{4} \gamma^{2}}$. This was illustrated on Figure 3.

\subsection{Regularity of the limit distribution}

In this section, we study the long term behavior of the drug concentration, that is, the distribution function of the $\operatorname{limit} C:=\lim _{t \rightarrow \infty} C(t)$, where the limit is taken in the sense of convergence in distribution.

Proposition 3. The random variable $C(t)$ converge in distribution, when t tends to infinity, to a well define random variable $C$ whose characteristic function is

$$
\varphi(\theta)=\exp \left\{\lambda \int_{0}^{1} \frac{\exp \left\{i \theta \alpha\left(u^{k_{e}}-u^{k_{a}}\right)\right\}-1}{u} d u\right\} .
$$

Proof. When $t$ tends to infinity, $\varphi_{t}$ tends pointwise to $\varphi$, which is continuous at $\theta=0$. By Lévy's theorem, this implies the result. 
Note that the distribution of $C$ invariant by time reversal: looking "backwards" in time, one see that $C(t)$ has the same law as

$$
C^{\prime}(t)=\frac{F D}{V_{d}} \frac{k_{a}}{k_{a}-k_{e}} \sum_{i}\left(e^{-k_{e} T_{i}}-e^{-k_{a} T_{i}}\right) \mathbb{1}_{\left(t \geq T_{i}\right)} ;
$$

as $t$ tend to infinity, the random variables $C^{\prime}(t)$ converge almost surely to

$$
C^{\prime}=\frac{F D}{V_{d}} \frac{k_{a}}{k_{a}-k_{e}} \sum_{i}\left(e^{-k_{e} T_{i}}-e^{-k_{a} T_{i}}\right),
$$

which therefore has the same distribution as $C$. In the sequel, we shall write

$$
C=\frac{F D}{V_{d}} \frac{k_{a}}{k_{a}-k_{e}} \sum_{i}\left(e^{-k_{e} T_{i}}-e^{-k_{a} T_{i}}\right),
$$

since we are only interested in distributional properties.

Proposition 4. The characteristic function $\varphi$ satisfies

$$
|\varphi(\theta)| \sim K|\theta|^{-\mu^{*}}, \quad \text { when } \quad \theta \rightarrow \infty,
$$

with $K$ a positive constant and $\mu^{*}=\frac{\lambda}{\left(k_{e} \wedge k_{a}\right)}=\max \left\{\mu_{e}, \mu_{a}\right\}$.

Proof. One computes:

$$
|\varphi(\theta)|=\exp \left\{-\lambda \int_{0}^{1} \frac{1-\cos \left(\theta \alpha\left(u^{k_{e}}-u^{k_{a}}\right)\right)}{u} d u\right\}=: \exp \{-\lambda I(\theta)\} .
$$

Set $0<\delta<1$, and decompose the integral $I(\theta)$ as follows:

$$
I(\theta)=I_{1}(\theta)-I_{2}(\theta)-\log (\delta),
$$

where

$$
\begin{aligned}
& I_{1}(\theta)=\int_{0}^{\delta} \frac{1-\cos \left(\theta \alpha\left(u^{k_{e}}-u^{k_{a}}\right)\right)}{u} d u, \\
& I_{2}(\theta)=\int_{\delta}^{1} \frac{\cos \left(\theta \alpha\left(u^{k_{e}}-u^{k_{a}}\right)\right)}{u} d u .
\end{aligned}
$$

When $0 \leq u \leq \delta$, we have that $\alpha\left(u^{k_{e}}-u^{k_{a}}\right) \sim|\alpha| u^{k_{e} \wedge k_{a}}$, and thus

$$
\begin{aligned}
I_{1}(\theta) & \sim \int_{0}^{\delta} \frac{1-\cos \left(\theta \alpha u^{k_{e} \wedge k_{a}}\right)}{u} d u \\
& \sim \frac{1}{k_{e} \wedge k_{a}} \log (\theta|\alpha|)+\frac{\gamma_{e}}{k_{e} \wedge k_{a}}+\log (\delta)+O\left(\frac{1}{\theta}\right),
\end{aligned}
$$


where $\gamma_{e}$ is the Euler constant.

Denote $h(u)=\alpha\left(u^{k_{e}}-u^{k_{a}}\right)$. The function $h$ has a global maximum at $u_{0}=\left(\frac{k_{e}}{k_{a}}\right)^{\frac{1}{k_{a}-k_{e}}}$ with $h "\left(u_{0}\right)<0$. Stationary phase arguments imply that, when $\theta$ tends to infinity,

$$
\begin{aligned}
I_{2}(\theta) & \sim \operatorname{Re}\left(\int_{\delta}^{1} \frac{e^{i \theta \alpha\left(u^{k_{e}}-u^{k_{a}}\right)}}{u} d u\right) \\
& \sim \operatorname{Re}\left(\frac{e^{i\left(\theta h\left(u_{0}\right)-\pi / 4\right) \sqrt{2 \pi}}}{u_{0} \sqrt{\theta\left|h^{\prime \prime}\left(u_{0}\right)\right|}}\right) \\
& =\frac{\cos \left(\theta h\left(u_{0}\right)-\pi / 4\right)}{u_{0}} \sqrt{\frac{2 \pi}{\theta\left|h^{\prime \prime}\left(u_{0}\right)\right|}} \\
& =O\left(\frac{1}{\sqrt{\theta}}\right) .
\end{aligned}
$$

Formulas (27), (28), (29) and (30) entail that

$$
I(\theta)=\frac{1}{k_{e} \wedge k_{a}} \log (\theta|\alpha|)+\frac{\gamma_{e}}{k_{e} \wedge k_{a}}+O\left(\frac{1}{\sqrt{\theta}}\right) .
$$

This implies that there exists $K>0$ such that $|\varphi(\theta)| \sim K|\theta|^{-\mu^{*}}$ when $\theta$ tend to infinity.

We denote by $F$ the probability distribution function of $C$, associated to $\varphi$. Then, from Proposition 4 we have the following results with respect to the regularity of $F$ :

1. $F$ has an $L^{2}$ density ${ }^{1}$ if and only if $\mu^{*}>\frac{1}{2}$.

2. For $\mu^{*}<1$ and $0<\varepsilon<1, \int_{1}^{\infty} \theta^{\mu^{*}-\varepsilon}|\varphi(\theta)| d \theta<\infty$ thus $F \in \operatorname{Lip}\left(\mu^{*}-\varepsilon\right)$.

3. For $\mu^{*}<\frac{1}{2}, \frac{1}{T} \int_{-T}^{T}|\varphi(\theta)|^{2} d \theta=\mathcal{O}\left(T^{-2 \mu^{*}}\right)$ thus $F \in \operatorname{Lip}\left(\mu^{*}\right)$.

4. A classical Tauberian theorem (see, e.g. [7], p.445) entails that:

$$
F(\varepsilon) \sim \frac{e^{-\mu^{*} \gamma_{e}}|\alpha|^{-\mu^{*}}}{\Gamma\left(\mu^{*}+1\right)}|\varepsilon|^{-\mu^{*}},
$$

when $\varepsilon \rightarrow 0$. Then, $F$ is not differentiable at 0 when $\mu^{*}<1$ and it has a finite non vanishing derivative at 0 exactly when $\mu^{*}=1$.

5. From Proposition 3 in [11], we have that, for any $x>0$,

$$
F(x+\varepsilon)-F(x)=\mathcal{O}(\varepsilon),
$$

when $\varepsilon \rightarrow 0^{+}$. This implies that 0 is the only possibly singular point of $F$.

\footnotetext{
${ }^{1}$ This means that the integral of the square of the probability density function is finite.
} 
The practical meaning of these results is that, when $\lambda<\min \left\{k_{a}, k_{e}\right\}$ the instant of intakes are too scarce, with respect to the absorption and the elimination rates, resulting in a high probability of having too small concentration of drugs.

\section{Continuous model with random intake instants and random doses}

In this section, we consider the generalization of the continuous model of Section 3 to allow for random doses. We study the main properties of the random concentration $C^{r d}(t)$, given by (16), which we recall here for convenience:

$$
C^{r d}(t)=\frac{F D}{V_{d}} \frac{k_{a}}{k_{a}-k_{e}} \sum_{i} D_{i}\left(e^{-k_{e}\left(t-T_{i}\right)}-e^{-k_{a}\left(t-T_{i}\right)}\right) 1_{\left(t \geq T_{i}\right)},
$$

where the $\left(T_{i}\right)_{i \in \mathbb{N}}$ again form a Poisson process, and the $\left(D_{i}\right)_{i \in \mathbb{N}}$ are random variables. $F$ and $V_{d}$ are constants. At time $T_{i}$, the dose taken is $D D_{i}$, and we assume that $\mathbb{E}\left(D_{i}\right)=1$, that $D_{i}$ is supported on $\mathbb{R}_{+}$, and that it has compact support (although we shall need only weaker assumptions).

The process $C^{r d}(t)$ thus defined is a marked Poisson process. In this work, we shall assume that the $\left(D_{i}\right)_{i \in \mathbb{N}}$ are independent and identically distributed random variables, where each $D_{i}$ may depend on $T_{i}$ but is independent from the $\left(T_{j}\right)_{j \neq i}$. This makes sense from a pharmacokinetic point of view, since it seems plausible that the patient will not adjust his dose at time $T_{i}$ on the basis of his past or future behavior except for the time lag from the previous take, although it would maybe be desirable to let $D_{i}$ depend also on $D_{i-1}$. We denote by $\nu(T,$.$) the conditional distribution of D_{i}$ knowing that $T_{i}=T$. Our assumptions allow to apply a generalized form of Campbell theorem [9] to the effect that the characteristic function $\varphi_{t}^{r d}$ of $C^{r d}(t)$ is given by:

$$
\varphi_{t}^{r d}(\theta)=\exp \left\{\lambda \int_{0}^{t} \int_{A}\left(e^{i \theta \alpha u h(t-x)}-1\right) \nu(x, d u) d x+i \theta \alpha h(t)\right\}
$$

where, as before, $\alpha=\frac{F D}{V_{d}} \frac{k_{a}}{k_{a}-k_{e}}, h(t)=e^{-k_{e} t}-e^{-k_{a} t}$ and $A$ is the support of the $D_{i}$.

\subsection{Variability of the concentration}

From (31), one deduces easily the mean and variance of $C^{r d}$ :

$$
\left(\varphi_{t}^{r d}\right)^{\prime}(\theta)=\varphi_{t}^{r d}(\theta)\left[\lambda \int_{0}^{t} \int_{A} i \alpha u h(t-x) e^{i \theta \alpha u h(t-x)} \nu(x, d u) d x+i \alpha h(t)\right],
$$

and thus

$$
\left(\varphi_{t}^{r d}\right)^{\prime}(0)=i \alpha\left(\mu_{e}\left(1-e^{-k_{e} t}\right)-\mu_{a}\left(1-e^{-k_{a} t}\right)\right) \mathbb{E}_{x}\left(D_{1}\right)+i \alpha\left(e^{-k_{e} t}-e^{-k_{a} t}\right) .
$$


where $\mathbb{E}_{x}\left(D_{1}\right)=\int_{A} u \nu(x, d u)$ is the expectation of $D_{i}$ knowing that $T_{i}=x$, which is equal to one for any $x$ by assumption. Thus we find that, not surprisingly:

$$
\mathbb{E}\left(C^{r d}(t)\right)=\mathbb{E}(C(t))
$$

Likewise,

$$
\begin{aligned}
\left(\varphi_{t}^{r d}\right) "(\theta)= & \varphi_{t}^{r d}(\theta)\left[\left(\lambda \int_{0}^{t} \int_{A} i \alpha u h(t-x) e^{i \theta \alpha u h(t-x)} \nu(x, d u) d x-\alpha^{2} h^{2}(t-x)\right)^{2}\right. \\
& \left.-\lambda \int_{0}^{t} \int_{A} \alpha^{2} u^{2} h^{2}(t-x) e^{i \theta \alpha u h(t-x)} \nu(x, d u) d x\right] .
\end{aligned}
$$

Using that, by definition, $\int_{A} u^{2} \nu(x, d u)=\mathbb{E}_{x}\left[D_{1}^{2}\right]$, this entails:

$$
\begin{aligned}
\left(\varphi_{t}^{r d}\right)^{\prime \prime}(0) & =-\mathbb{E}[C(t)]^{2}-\lambda \alpha^{2} \int_{0}^{t} h^{2}(t-x) \int_{A} u^{2} \nu(x, d u) d x \\
& =-\mathbb{E}[C(t)]^{2}-\lambda \alpha^{2} \int_{0}^{t} h^{2}(t-x) \mathbb{E}_{x}\left(D_{1}^{2}\right) d x
\end{aligned}
$$

or, since $\left(\varphi_{t}^{r d}\right)^{\prime \prime}(0)=-\mathbb{E}[C(t)]^{2}-\operatorname{Var}\left(C^{r d}(t)\right)$,

$$
\operatorname{Var}\left(C^{r d}(t)\right)=\lambda \alpha^{2} \int_{0}^{t} h^{2}(t-x) \mathbb{E}_{x}\left[D_{1}^{2}\right] d x .
$$

However,

$$
\mathbb{E}_{x}\left(D_{1}^{2}\right)=\operatorname{Var}_{x}\left(D_{1}\right)+\mathbb{E}_{x}\left[D_{1}\right]^{2}=\operatorname{Var}_{x}\left(D_{1}\right)+1
$$

( $\operatorname{Var}_{x}\left(D_{1}\right)$ denotes the variance of $D_{i}$ knowing that $T_{i}=x$ ) and

$$
\lambda \alpha^{2} \int_{0}^{t} h^{2}(t-x) d x=\operatorname{Var}(C(t))
$$

thus

$$
\operatorname{Var}\left(C^{r d}(t)\right)=\operatorname{Var}(C(t))+\lambda \alpha^{2} \int_{0}^{t} h^{2}\left(t_{x}\right) \operatorname{Var}_{x}\left(D_{1}\right) d x .
$$

Assuming that $\operatorname{Var}_{x}\left(D_{1}\right)=\operatorname{Var}\left(D_{1}\right)$, i.e. the variance of $D_{i}$ does not depend on the value of $T_{i}$, one gets:

$$
\operatorname{Var}\left(C^{r d}(t)\right)=\mathbb{E}\left[D_{1}^{2}\right] \operatorname{Var}(C(t))=\left(1+\operatorname{Var}\left(D_{1}\right)\right) \operatorname{Var}(C(t)) .
$$

Thus random-dosing results in multiplying the variance of the deterministic-dose case by $\mathbb{E}\left(D_{1}^{2}\right)$. It is then easy to obtain inequalities similar to (22), (23) and (24) for the randomdose case.

Two particular cases may be of special interest: 
- Discrete distribution: the $D_{i}$ assume the values $\left\{d_{1}, \ldots, d_{m}\right\} \subset \mathbb{R}_{+}$with probabilities $q_{1}, \ldots, q_{m}$, independently of the $T_{i}$. We denote by $C_{t}^{r d, d}$ and $\varphi_{t}^{r d, d}$ the concentration and characteristic function. Then:

$$
\varphi_{t}^{r d, d}(\theta)=\prod_{j=1}^{m} \varphi_{t}\left(q_{j} \lambda, d_{j} \theta\right)
$$

where we have written $\varphi_{t}\left(q_{j} \lambda, \cdot\right)$ instead of $\varphi_{t}($.$) for the characteristic function in the$ deterministic-dose case, given in equation (19), but with parameter $q_{j} \lambda$ instead of $\lambda$. This equality also holds when $t$ tends to infinity, yielding the long term concentration characteristic function. The variance is given by:

$$
\operatorname{Var}\left(C^{r d, d}(t)\right)=\mathbb{E}\left[D_{1}^{2}\right] \operatorname{Var}(C(t))=\left(\sum_{j=1}^{m} q_{j} d_{j}^{2}\right) \operatorname{Var}(C(t)) .
$$

It is of interest to characterize the situation giving the largest variance among all admissible random dosings with arbitrary $m, q_{i}$, and $d_{i}$. In other words, we look for the value of $V_{\max }:=\max \left\{\operatorname{Var}\left(C^{r d, d}(t)\right)\right\}$ subject to $m>1,\left(q_{1}, \ldots, q_{m}\right) \in[0,1]^{m}$ with at least two $q_{i}$ non zero and $\sum_{j=1}^{m} q_{i}=1,\left(d_{1}, \ldots, d_{m}\right) \in[a, b]$ with $b>a>0$, and $\sum_{j=1}^{m} q_{i} d_{i}=1$. It is easily shown, for instance using Lagrange multipliers, that the maximum is reached for $m=2$ and $d_{1}=a, d_{2}=b$. In this case,

$$
V_{\max }=(a+b-a b) \operatorname{Var}(C(t)) .
$$

Thus the worst-case situation is when the patient "oscillates" between two dosings whose average is the prescribed one.

- Uniform distribution: the $D_{i}$ are uniformly distributed over $[a, b] \subset \mathbb{R}_{+}$, independently of the $T_{i}$. We denote by $C_{t}^{r d, u}$ and $\varphi_{t}^{r d, u}$ the concentration and characteristic function. In this case, one computes:

$$
\varphi_{t}^{r d, u}(\theta)=\exp \left\{\frac{\lambda}{b-a} \int_{a}^{b} \int_{e^{-t}}^{1} \frac{e^{i \theta y \alpha\left(u^{k_{e}}-u^{k_{a}}\right)}-1}{u} d u d y+i \theta \alpha h(t)\right\} .
$$

This last function is easily seen to be convergent when $t$ tends to infinity, which gives us the long term concentration characteristic function. Note that $\varphi^{r d, u}$ tends to $\varphi$ when the couple $(a, b)$ tends to $(D, D)$ : the concentration with random doses uniformly distributed on $[a, b]$ tends in law to the concentration with fixed dose $D$. The variance is given by:

$$
\operatorname{Var}\left(C^{u}(t)\right)=\mathbb{E}\left(D_{1}^{2}\right) \operatorname{Var}(C(t))=\frac{a^{2}+a b+b^{2}}{3} \operatorname{Var}(C(t)) .
$$

We can see that the choice of $[a, b]$ maximizing the variance under the constraint $\mathbb{E}\left(D_{1}\right)=1$ is $a=0, b=2$, as expected. 


\subsection{Regularity of the limit distribution}

We first consider the two cases of discrete and uniform random dosing.

- Discrete distribution

From (32) and Proposition 4, one easily sees that:

$$
\left|\varphi^{r d, d}(\theta)\right| \sim \prod_{j=1}^{m}\left(d_{j} \theta\right)^{-q_{j} \mu^{*}}=K \theta^{-\mu^{*}}
$$

when $\theta$ tends to infinity, where $K$ is a constant.

- Uniform distribution

In this case, the limit of the function $\varphi_{t}^{r d, u}$, defined in Formula (34), when $t$ tends to infinity is:

$$
\varphi^{r d, u}(\theta)=\exp \left\{\frac{\lambda}{b-a} \int_{a}^{b} \int_{0}^{1} \frac{e^{i \theta y \alpha\left(u^{k_{e}}-u^{k_{a}}\right)}-1}{u} d u d y\right\} .
$$

The modulus of $\varphi^{r d, u}$ is

$$
\begin{aligned}
\left|\varphi^{r d, u}(\theta)\right| & =\exp \left\{\frac{-\lambda}{(b-a)} \int_{a}^{b} \int_{0}^{1} \frac{1-\cos \left(\theta y \alpha\left(u^{k_{e}}-u^{k_{a}}\right)\right)}{u} d u d y\right\}, \\
& =\exp \left\{\frac{-\lambda}{(b-a)} \int_{a}^{b} I(\theta y) d y\right\} .
\end{aligned}
$$

The proof of Proposition 4 shows that, for large enough $\theta$,

$$
I(\theta y) \sim \frac{1}{k_{e} \wedge k_{a}} \log (\theta y|\alpha|)+\text { constant } .
$$

This implies that there exists $K>0$ such that $|\varphi(\theta)| \sim K|\theta|^{-\mu^{*}}$ when $\theta$ tends to infinity. This fact will be justified in the proof of Proposition 5.

Thus in both particular cases above, we recover the same behaviour for the characteristic function as in the deterministic-dose case. As the next proposition shows, this is in fact a general feature of all random dosings provided $\nu(x, d u)$ does not depend on $x$, with an additional mild condition:

Proposition 5. Assume that $\nu(x, d u)=\nu(d u)$ and that $\int_{A} \log (y) \nu(d y)<\infty$. Then

$$
\left|\varphi^{r d}(\theta)\right| \sim K|\theta|^{-\mu^{*}}
$$

when $\theta$ tends to infinity, where $K$ is a positive constant. 
Proof. Thanks to the assumption on $\nu$, one computes

$$
\begin{aligned}
\left|\varphi^{r d, u}(\theta)\right| & =\exp \left\{-\lambda \int_{A} \int_{0}^{1} \frac{1-\cos \left(\theta y \alpha\left(u^{k_{e}}-u^{k_{a}}\right)\right)}{u} d u \nu(d y)\right\} \\
& =\exp \left\{-\lambda \int_{A} I(\theta y) \nu(d y)\right\} .
\end{aligned}
$$

The proof of Proposition 4 shows that, when $\theta$ tends to infinity,

$$
-\lambda I(\theta y) \sim K-\mu^{*}(\log (\theta)+\log (y))
$$

for a certain constant $K$, with in addition $-\lambda I(\theta y)-K+\mu^{*}(\log (\theta)+\log (u))$ tending to 0 with a rate of convergence $\frac{1}{\sqrt{\theta}}$. Thus, using the assumption on the logarithmic moment of $\nu$,

$$
-\lambda \int_{A} I(\theta y) \nu(d y) \sim K-\mu^{*} \log (\theta)-\mu^{*} \int_{A} \log (y) \nu(d y)
$$

and one finishes up the proof with the help of a dominated convergence argument to show that the difference between the right-hand side and the left-hand side in the equivalent above indeed tends to 0 when $\theta$ tends to infinity.

Proposition 5 shows that random dosing, at least when the distribution of the $D_{i}$ is independent of the $T_{i}$, does not alter the regularity of the distribution of the long-term concentration as compared to the deterministic-dose case.

\section{Discrete model with random intake instants and deterministic doses}

We study in this section a discretization in time of the model above. In other words, instead of taking the drug at arbitrary time instants $t \in R^{+}$, we assume that the patient will only do so at (random) times which form a subset of $\left\{t_{l}^{n}=l / n ; l \in \mathbb{N}\right\}$, where $n$ is a fixed number. We shall first rewrite the drug concentration in this discrete setting. It will appear that the discretized concentration $C_{d}$ has the same law as an object that has been thoroughly studied in mathematics under the name of infinite Bernoulli convolution. We will study the variability of the discretized concentration. Then we will show that, when $n$ tends to infinity, the discretized model indeed tends distribution-wise to the continuous-time one. We will finally study the regularity of the long term behaviour of the discretized concentration for $n$ fixed or tending to infinity, and show that it is, under certain circumstances, singular.

\subsection{The discretized concentration}

The discretized model taking the following form. Let $h=\frac{1}{n}$ be the discretization step. Thus, the drug intakes can only occur at times $t_{j}=j h$ with $j \in \mathbb{N}$. 
From a general point of view, the discrete analog of the Poisson process is the Bernoulli process. Indeed, in the continuous framework, the Poisson process is the only counting process which has stationary and independent increments. Likewise, the only discrete counting process with the same property is the Bernoulli process. In terms of waiting times, this amounts to replacing the i.i.d., memoryless, exponential random variables $S_{n}=T_{n+1}-T_{n}$, by i.i.d. random variables following a geometric distribution (recall that the geometric distribution is the only memoryless discrete distribution).

We are thus led to consider a sequence $\left(X_{j}\right)_{j \in \mathbb{N}}$ of i.i.d. Bernoulli random variables with parameter $p$ such that $X_{j}=1$ if the patient takes the drug at time $t_{j}$ and $X_{j}=0$ if not.

In this discrete model the number of drug intakes per unite time is $\frac{p}{h}$, so one has choose $p=\lambda h$. Note that, for the model to make sense, $p$ must be smaller than one, which translates into $\lambda h<1$.

At a fixed time $t_{n}$ the contribution of the $j$-th intake to the current concentration is

$$
\alpha\left(\beta_{e}^{n-j}-\beta_{a}^{n-j}\right) X_{j},
$$

where $\beta_{e}=e^{-k_{e} h}$ and $\beta_{a}=e^{-k_{a} h}: \beta_{e}$ is the elimination rate for one time step and $\beta_{a}$ is the absorption rate for one time step.

Thus, the total concentration at time $t_{n}$ is given by

$$
C_{n}=\alpha \sum_{j=0}^{n}\left(\beta_{e}^{n-j}-\beta_{a}^{n-j}\right) X_{j} .
$$

Since the random variable $\left(X_{j}\right)_{j \in \mathbb{N}}$ are independent, they are exchangeable, and in particular the vector $\left(X_{0}, \ldots, X_{n}\right)$ has the same distribution as the reversed vector $\left(X_{n}, \ldots, X_{0}\right)$. Hence $C_{n}$ is equal in distribution to

$$
Y_{n}=\alpha \sum_{j=0}^{n}\left(\beta_{e}^{j}-\beta_{a}^{j}\right) X_{j} .
$$

The sequence $\left(Y_{n}\right)$ converges almost surely to the random variable $Y_{(p)}$, given by equation (17) in Section 2.5; i.e.,

$$
Y_{(p)}=\alpha \sum_{j=0}^{\infty}\left(\beta_{e}^{j}-\beta_{a}^{j}\right) X_{j} .
$$

The distribution of $Y_{(p)}$ is thus the one of the long term concentration in this model. Since $Y_{(p)}$ is an infinite sum of independent Bernoulli random variables, its law is an infinite convolution of Bernoulli distribution, hence its name "infinite Bernoulli convolution".

From the independence of $\left(X_{j}\right)_{j \in \mathbb{N}}$ we have that the characteristic function of $Y_{(p)}$ is given by

$$
\varphi_{p}(\theta)=\prod_{j=0}^{\infty}\left[(1-p)+p e^{i \theta \alpha\left(\beta_{e}^{j}-\beta_{a}^{j}\right)}\right] .
$$




\subsection{Variability of the discretized concentration}

One computes easily the mean $E_{d i s c}$ and variance $V a r_{d i s c}$ in the discretized model:

$$
\begin{aligned}
E_{\text {disc }} & :=\mathbb{E}\left[Y_{(p)}\right]=\alpha p\left(\frac{1}{1-\beta_{e}}-\frac{1}{1-\beta_{a}}\right), \\
\operatorname{Var}_{\text {disc }} & :=\operatorname{Var}\left(Y_{(p)}\right)=\alpha^{2} p(1-p)\left(\frac{1}{1-\beta_{e}^{2}}-\frac{2}{1-\beta_{e} \beta_{a}}+\frac{1}{1-\beta_{a}^{2}}\right) .
\end{aligned}
$$

As we shall see in the next section, the discrete model tends in law to the continuous one when $p$ tends to 0 . For now, we simply remark that, indeed, $E_{d i s c}$ tends to $\mu_{e} \frac{F D}{V_{d}}$ and $\operatorname{Var}_{\text {disc }}$ tends to $\frac{\mu_{e}}{2} \frac{1}{1+r}\left(\frac{F D}{V_{d}}\right)^{2}$ when $p$ tends to 0 , i.e. the mean and variance of (20) and (21) (note that, when $p$ tends to $0, \beta_{e} \sim 1-\frac{p}{\mu_{e}}$ and $\beta_{a} \sim 1-\frac{p}{\mu_{a}}$ so $\beta_{e}$ and $\beta_{a}$ tend to 1 ).

For $E_{d i s c}$ constant, say $E_{d i s c}=1$, the variance in the discrete model reads:

$$
\operatorname{Var}_{d i s c}=\frac{1-p}{p} \frac{\left(1-\beta_{e}\right)\left(1-\beta_{a}\right)\left(1+\beta_{e} \beta_{a}\right)}{\left(1+\beta_{e}\right)\left(1+\beta_{a}\right)\left(1-\beta_{e} \beta_{a}\right)} .
$$

For a fixed $p$, we see that $V a r_{d i s c}$ tends to 0 at the speed $\frac{1-p}{2 \mu_{e}}$ when $\mu_{e}$ tends to infinity, which is the same rate as $V a r$, the variance in the continuous random model of Section 3.1. When $\mu_{e}$ tends to $0, \operatorname{Var}_{\text {disc }}$ tends to $\frac{1-p}{p} \frac{1-\beta_{a}}{1+\beta_{a}}$ in contrast to both the deterministic and random continuous cases, where the variance tends to infinity. We show on Figure 10 the behaviour of the ratio $V a r_{d i s c} / V a r$ as a function of $\mu_{e}$ and $\mu_{a}$ when the mean is kept constant.

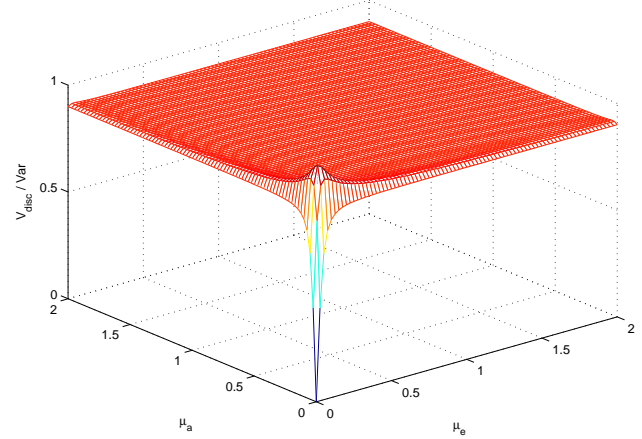

Figure 10: Ratio $\operatorname{Var}_{d i s c} / \operatorname{Var}$ as a function of $\mu_{e}$ and $\mu_{a}$ when the mean is kept constant.

As a consequence of Chebychev inequality we have (compare with Formulas (24) and (25)):

$$
\mathbb{P}(|C-\mathbb{E}[C]| \geq \gamma E[C]) \leq \frac{1}{\gamma^{2}} \frac{1}{1+r}+\frac{2}{1-r^{2}} \frac{1-p}{p} \frac{\left(1-\beta_{e}\right)\left(1-\beta_{a}\right)\left(1+\beta_{e} \beta_{a}\right)}{\left(1+\beta_{e}\right)\left(1+\beta_{a}\right)\left(1-\beta_{e} \beta_{a}\right)} .
$$




\subsection{Limit when the discretization step tends to 0}

The following proposition describes the behaviour of $Y_{(p)}$ when $n$ tends to infinity or, which is the same, when $p$ tends to zero. Note that this also equivalent to letting $\beta_{e}$ and $\beta_{a}$ tend to 1.

Proposition 6. $Y_{(p)}$ converges in law to $C$ when $p$ tends to zero.

Proof. The proof is analogous to the one of Proposition 4 in [11]: one shows that the characteristic function $\varphi_{p}$ of $Y_{(p)}$ tends pointwise to the characteristic function $\varphi$ of $C$. The details are omitted.

In practical terms, this results means that, as long as we are only interested in distributional properties of the concentration, we may consider the discrete model instead of the continuous one provided $p$ is chosen small enough. Note however that the discrete model with arbitrary value of $p$ is interesting in its own right.

\subsection{Regularity of the discretized model}

We assume that $\mu_{a} \neq \mu_{e}$, since $Y_{(p)}$ is constantly equal to zero when $\mu_{a}=\mu_{e}$. The work [1] provides an analysis of the regularity of generalized infinite Bernoulli convolutions, and specially its fractal properties. The interested reader may also consult $[6,16,17]$ for studies on regular infinite Bernoulli convolutions.

The relevant parameter here is $\beta^{*}=\beta_{e} \vee \beta_{a}$. The results of [1] show that the regularity of the discretized model is very different depending on whether $\beta^{*}<\frac{1}{2}$ or $\beta^{*} \geq \frac{1}{2}$ :

- When $\beta^{*}<\frac{1}{2}$, the distribution of $Y_{(p)}$ is singular with respect to the Lebesgue measure. More precisely, the Hausdorff dimension of its support is equal to

$$
\liminf _{n \rightarrow \infty} \frac{-n \log (2)}{\log \left(\rho_{n}\right)}=\frac{\log (2)}{\log \left(\beta^{*}\right)}<1,
$$

where $\rho_{n}=\alpha\left(\frac{\beta_{e}^{n+1}}{1-\beta_{e}}-\frac{\beta_{a}^{n+1}}{1-\beta_{a}}\right)$. The Hausdorff dimension measures the "size", in a certain sense, of the support of the measure, i.e. where it is concentrated. A Hausdorff dimension smaller than one means that the set of possible values taken by the concentration is extremely sparse and does not form a continuum. In addition, the probability of being in an interval varies in a very non-smooth way with the bounds of the interval. In practical terms, this means that probability distribution of the concentration will be highly irregular : it will vary erratically, taking only very particular values, and the probability that it ranges in some interval may vary wildly when the interval changes. Note that $\beta^{*}<\frac{1}{2}$ is equivalent to $p>\mu^{*} \log (2)$ or $h k^{*}>\log (2)$ with $k^{*}=k_{e} \wedge k_{a}$, (recall that $h$ is the discretization step ). This reflects the fact that, for fixed $k^{*}$, the time instants at which the patient is supposed to take his drugs are sufficiently spaced, and that he forgets to do so randomly at some of these instants. 
Alternatively, for a fixed $h, k^{*}$ must be sufficiently large. Note also that, in view of $p<1$, this is possible only if $\mu^{*}<\frac{1}{\log (2)}$.

- The case $\beta^{*}>\frac{1}{2}$ is mathematically much more delicate and is not completely settled. Results given in $[5,15]$ entail that the distribution of $Y_{(p)}$ is absolutely continuous for Lebesgue-almost all $\beta^{*}$ in $\left(\frac{1}{2}, 1\right)$ when $p=\frac{1}{2}$. This means that the distribution of the concentration has a probability density: in other words, in contrast with the case $\beta^{*}<\frac{1}{2}$, we are in the usual situation where the probability that the concentration ranges in some interval varies smoothly when the interval changes.

- In all other cases, the characterization of the regularity of the distribution of $Y_{(p)}$ remains an open problem.

\section{Discrete model with random intake instants and random doses}

In the discrete case, the random-dose model takes the following form:

- We still have a sequence of i.i.d. Bernoulli random variables $\left(X_{j}\right)_{j \geq 0}$ with parameter $p$, which mark the random instants of drug intake.

- To account for the random dosing, we consider a sequence of i.i.d. random variables $\left(D_{j}\right)_{j \geq 0}$, that will represent the doses, with distribution $\nu$ compactly supported on $\mathbb{R}_{+}^{*}$. We will let $\bar{\nu}$ denote the product measure $\nu \otimes \mathbb{N}$.

- We make the assumption $\mathbb{E}\left[D_{1}\right]=1$, which means that the patient takes the normal dose on average.

- We suppose that all the $D_{i}$ are independent of the $X_{j}$.

- As before, $\beta_{e}=e^{-k_{e} h}$ and $\beta_{a}=e^{-k_{a} h}$ are respectively the elimination rate and the absorption rate for one time step.

We investigate the behaviour of the almost sure limit (with respect to $\bar{\nu}$ ) of the steady state concentration $Y_{(p)}^{r d}$, given by Formula (18) in Section 2.6, i.e.

$$
Y_{(p)}^{r d}=\alpha \sum_{j=0}^{\infty}\left(\beta_{e}^{j}-\beta_{a}^{j}\right) D_{j} X_{j} .
$$

Independence of $\left(X_{j}\right)_{j \in \mathbb{N}}$ and $\left(D_{j}\right)_{j \in \mathbb{N}}$ entail that the characteristic function of $Y_{(p)}^{r d}$ is given by

$$
\varphi_{p}^{r d}(\theta)=\prod_{j=0}^{\infty}\left[(1-p)+p \int e^{i \theta \alpha\left(\beta_{e}^{j}-\beta_{a}^{j}\right) u} \nu(d u)\right] .
$$

As before, we first proceed to investigate the variability in this model, and then the limit when the discretization step tends to zero. We do not characterize the regularity of the concentration, as no results are available in this more complex situation. 


\subsection{Variability of the concentration}

The mean $E_{d i s c}^{r d}$ and variance $V a r_{d i s c}^{r d}$ in the random-dose discretized model are easily seen to be:

$$
\begin{aligned}
E_{d i s c}^{r d} & :=\mathbb{E}\left[Y_{(p)}^{r d}\right]=\alpha p\left(\frac{1}{1-\beta_{e}}-\frac{1}{1-\beta_{a}}\right)=E_{\text {disc }}, \\
\operatorname{Var}_{\text {disc }}^{r d} & :=\operatorname{Var}\left(Y_{(p)}^{r d}\right)=\alpha^{2}\left(\operatorname{Var}\left(D_{1}\right) p+p(1-p)\right)\left(\frac{1}{1-\beta_{e}^{2}}-\frac{2}{1-\beta_{e} \beta_{a}}+\frac{1}{1-\beta_{a}^{2}}\right) \\
& =\left(\frac{\operatorname{Var}\left(D_{1}\right)}{1-p}+1\right) \operatorname{Var}_{\text {disc }} .
\end{aligned}
$$

Random-dosing thus results in multiplying the variance of the deterministic-dose discretized model by $\frac{\operatorname{Var}\left(D_{1}\right)}{1-p}+1$. It is then easy to study the variability of the random-dose discretized model using the analysis developed in Section 5.2 for the discretized model.

\subsection{Limit when the discretization step tends to 0}

The following result can be proved in the same way as Proposition 4 in [11]:

Proposition 7. $Y_{(p)}$ converges in law to $C^{\text {rd }}$ when $p$ tends to zero.

Again, this means that, if one is only interested in distributional properties of the concentration, the discrete model may be considered in place of the continuous one provided $p$ is chosen small enough.

\section{Acknowledgements}

The research of L.J. Fermín was supported by Proyect DIGITEO DIM, ANIFRAC : Uncertaities in processes with fractal characteristics, and by a research grant from the Proyect DIUV N ${ }^{\circ} 2 / 2011$ of the University of Valparaíso.

\section{References}

[1] Albeverio, S., Torbin, G. (2008). On fine fractal properties of generalized infinite Bernoulli convolutions. Bull.Sci. Math. 132: 711-727.

[2] Barrière, O., Jun, L., Fahima, N. (2011). A Bayesian approach for the estimation of patient compliance based on the last sampling information. Journal of Pharmacokinetics and Pharmacodynamics 38 (3):333-351. 
[3] Barrière, O., Jun, L., Fahima, N. (2013). Compliance spectrum as a drug fingerprint of drug intake and drug disposition. Journal of Pharmacokinetics and Pharmacodynamics 40 (1): 41-52.

[4] Fermin, L., Lévy Véhel, J. Modeling patient poor compliance in the multi-IV administration case with Piecewise Deterministic Markov Models, preprint.

[5] Cooper, M. (1998). Dimension, measure and infinite Bernoulli convolutions, Math. Proc. Cambr. Phil. Soc. 124: 135-149.

[6] Erdós, P. (1939). On a family of symmetric Bernoulli convolutions, Amer. J. Math. 61: 974-975.

[7] Feller, W. (1971). An introduction to probability theory and its applications, Vol. II, 3rd edition, Wiley.

[8] Gaudreault, F., Drolet, P., Fallaha, M. and Varin, F. (2012). A population pharmacokinetic model for the complex systemic absorption of ropivacaine after femoral nerve block in patients undergoing knee surgery. Journal of Pharmacokinetics and Pharmacodynamics. 39 (6): 635-642.

[9] Kingman, J.F.C. (1993). Poisson Processes, Oxford University Press, Oxford.

[10] Iskedjian, M., Einarson, T.R., MacKeigan, L.D., Shear, N., Addis, A., Mittmann, N., and Ilersich, A.L. (2002). Relationship between daily dose frequency and adherence to antihypertensive pharmacotherapy: evidence from a meta-analysis. Clin. Ther. 24 (2), 302-316.

[11] Lévy Véhel, P.E, Lévy Véhel, J. (2012). Variability and singularity arising from poor compliance in a pharmacokinetic model I: the multi-IV case. Journal of Pharmacokinetics and Pharmacodynamics 40 (1):15-39.

[12] Loeve, M.(1977). Probability Theory I, Fourth Edition, Springer Verlag.

[13] Li, J. and Nekka, F. (2006). A Pharmacokinetic Formalism Explicitly Integrating the Patient Drug Compliance. J. Pharmacokinet. Pharmacodyn. 34 (1), 115-139.

[14] Li, J. and Nekka, F. (2009). A probabilistic approach for the evaluation of pharmacological effect induced by patient irregular drug intake. J. Pharmacokinet. Pharmacodyn. 36: 221-238.

[15] Pratsiovytyi, M. and Torbin, G. (1998). A class of random variables of the Jessen-Wintner type. Proceedings of the Ukrainian National Academy of Sciences (Dopovidi Nat. Acad. Nauk). 4: 48-54.

[16] Peres, Y., Schlag, W., Solomyak, B. (2000). Sixty years of Bernoulli convolutions. Fractal geometry and stochastics, II (Greifswald/Koserow, 1998), Progr. Probab., Birkhäuser, Basel, 46: 39-65.

[17] Peres, Y. and Solomyak, B. (1996). Absolute Continuity of Bernoulli Convolution, a Simple Proof. Math. Res. Lett. 3 (2): 231-239. 
[18] Sheiner L.B., Rosenberg B., Marathe V.V. (1977). Estimation of population characteristics of pharmacokinetic parameters from routine clinical data. J. Pharmacokinet Biopharm. 5 (5):445-79.

[19] Widmer, N. et al. (2006). Population pharmacokinetics of imatinib and the role of a1-acid glycoprotein. British Journal of Clinical Pharmacology. 62 (1):97-112. 